\title{
An Empirical Study of Transaction Throughput Thrashing Across Multiple Relational DBMSes
}

\author{
Young-Kyoon Suh ${ }^{\mathrm{a}, 1, *}$, Richard T. Snodgrass ${ }^{\mathrm{b}}$, Sabah Currim ${ }^{\mathrm{c}}$ \\ ${ }^{a}$ Supercomputing RED Center, KISTI, Daejeon, Republic of Korea, 34141 \\ ${ }^{b}$ Department of Computer Science, University of Arizona, Tucson, Arizona, 85721 \\ ${ }^{c}$ Alumni Association, University of Arizona, Tucson, Arizona, 85721
}

\begin{abstract}
Modern DBMSes are designed to support many transactions running simultaneously. DBMS thrashing is indicated by the existence of a sharp drop in transaction throughput. Thrashing behavior in DBMSes is a serious concern to database administrators (DBAs) as well as to DBMS implementers. From an engineering perspective, therefore, it is of critical importance to understand the causal factors of DBMS thrashing. However, understanding the origin of thrashing in modern DBMSes is challenging, due to many factors that may interact with each other.

This article aims to better understand the thrashing phenomenon across multiple DBMSes. We identify some of the underlying causes of DBMS thrashing. We then propose a novel structural causal model to explicate the relationships between various factors contributing to DBMS thrashing. Our model derives a number of specific hypotheses to be subsequently tested across DBMSes, providing empirical support for this model as well as important engineering implications for improvements in transaction processing.
\end{abstract}

Keywords: DBMS Thrashing, Transaction, Throughput, Factors, Structural Causal Model, Empirical Study

\footnotetext{
*Corresponding author

Email addresses: ${ }^{a}$ youngkyoon.suh@gmail.com (Young-Kyoon Suh), ${ }^{b}$ rts@cs.arizona.edu (Richard T. Snodgrass), ${ }^{c}$ scurrim@email.arizona.edu (Sabah Currim)

${ }^{1}$ The author was at the University of Arizona when starting this research.
} 


\section{Introduction}

Database management systems (DBMSes) are a core component of current information technology (IT) systems [1]. DBMSes have been widely adopted to serve a variety of workloads such as on-line analytical processing (OLAP) [2] and on-line transaction processing (OLTP) [3] applications. Over the last five decades, achieving high performance in handling workloads has been a primary goal in the database community. Accordingly, various methodologies and techniques have been proposed to enhance the efficiency of DBMSes and thereby, of database applications.

Many DBMS performance issues have been addressed and resolved over the decades, but scalability is still regarded as a major concern $[1,4]$. When a scalability bottleneck is encountered in a DBMS, transaction throughput can drop. In the worst case the DBMS may experience performance degradation exhibited by thrashing [5], in which a drastic reduction in throughput occurs.

\subsection{The DBMS Thrashing Problem}

DBMS thrashing $[6,7,8,9]$ is a precipitous drop of transaction throughput over increasing multiprogramming level (MPL), defined as the number of active database connections. Interestingly, DBMS thrashing is observed in modern high-performance DBMSes, as demonstrated in Figure 1.

We studied three proprietary DBMSes ${ }^{2}$ (denoted by $X, Y$, and $Z$ ) and two open-source DBMSes (MySQL and PostgreSQL). Each DBMS was run exclusively on a dedicated machine with the same hardware specification as will be described in Table 2 .

Note that the configurations differ across the DBMSes in Figure 1, specifically, in the number of processors (numProcs) on each machine running a DBMS, as our goal in these initial experiments are just to see whether each DBMS can be induced to thrash. This variation may explain why PostgreSQL's (Figure 1(e)) performance appeared to be about two times better than that of MySQL (Figure 1(d)), primarily because of doubling of the number of processors. It may also account for why DBMS $Y$ 's (Figure 1(b)) performance appeared to dominate the others by up to about ten times, perhaps due to the most eight processors. Moreover, that ten times more tuples were scanned compared to the others, may explicate why the

\footnotetext{
${ }^{2}$ As legal agreements do not allow us to report the performance evaluation results of those proprietary DBMSes, their names are anonymized in Figure 1.
} 


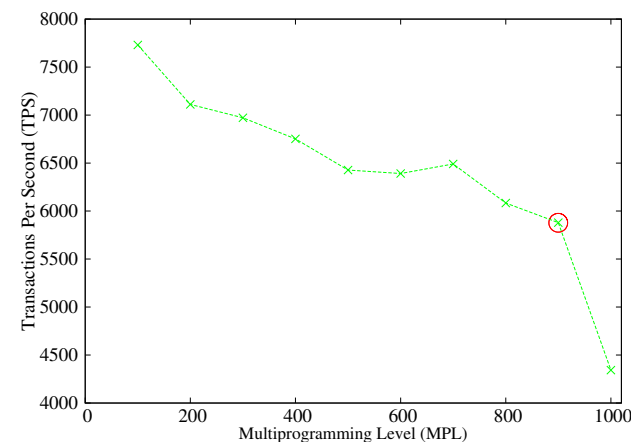

(a) DBMS $X$ 's Thrashing (numProcs=1)

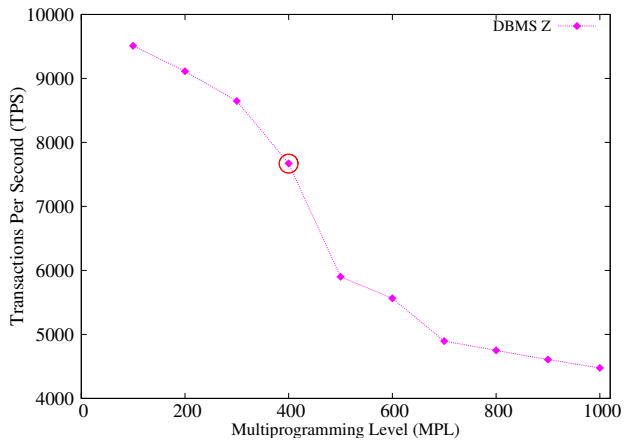

(c) DBMS Z's Thrashing (numProcs=8, $10 \%$ more tuples scanned)

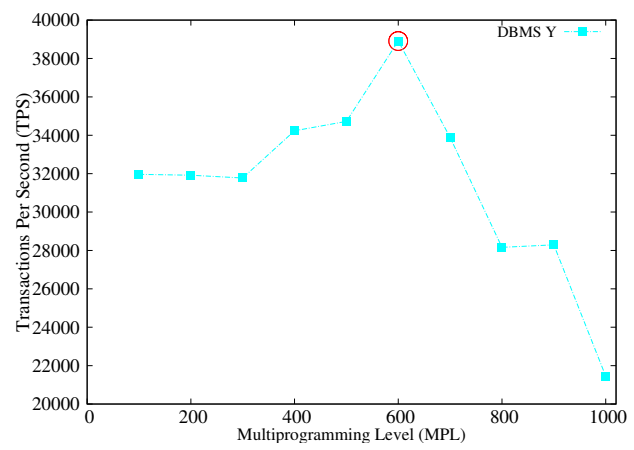

(b) DBMS Y's Thrashing (numProcs=8)

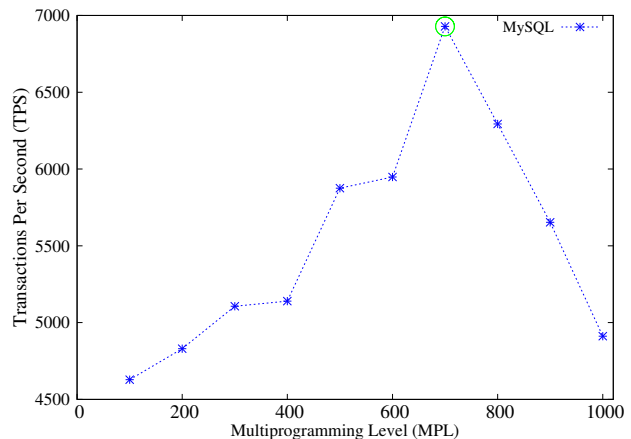

(d) MySQL's Thrashing (numProcs=2)

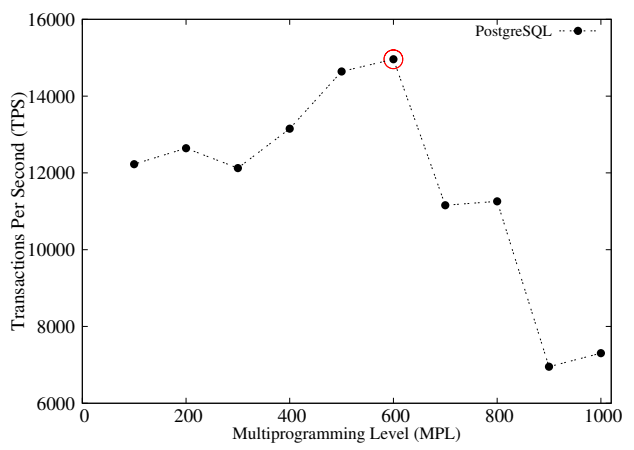

(e) PostgreSQL's Thrashing (numProcs $=4$ )

Figure 1: Observed DBMS Thrashing 
performance of DBMS $Z$ was poorer than that of DBMS $Y$ 's given the same number of processors. That said, a focus on these experiments was not to see "different performance" under different configuration across the DBMSes. The real attention was on "something else," i.e., thrashing, to be discussed now.

In the figure the $x$-axis shows varying MPL from 100 to 1,000 in steps of 100, and the $y$-axis represents the number of completed transactions, also known as transactions per second (TPS)[10], that we observed for a run at each MPL. The transactions in Figure 1 are all read-only, with the range of selected rows overlapping between the transactions within the same MPL. (We will describe how we generate transactions in greater detail in Section 5.)

As shown in Figure 1, we observed that thrashing occurred in all the DBMSes. For DBMS $X$, thrashing started at an MPL of 900, marked with a red circle. At that MPL, there is a sharp drop in throughput, indicating that DBMS $X$ entered the "thrashing phase." The MPL of 900 is thus called the "thrashing point," after which DBMS $X$ experienced thrashing.

The figure shows that this thrashing phenomenon was also observed in the other DBMSes, whose thrashing occurred at MPLs of 600,400, 700, and 600 marked with a red circle. In particular, there were slight bumps at MPLs of 900 and 800 (and 1000) after DBMS Y's and PostgreSQL's thrashing occurred. But such bumps were within the thrashing phases of those two DBMSes: The thrashing phase persisted until the last MPL of 1,000.

The experiments actually demonstrate two things. One is that thrashing occurs in modern relational DBMSes. Second is that this thrashing phenomenon is observed across all the DBMSes studied.

Thrashing behavior in DBMSes is a serious concern to DBAs (database administrators) engaged in developing OLTP or OLAP systems, as well as DBMS implementers developing technologies related to concurrency control. In general, if thrashing occurs in a DBMS, many transactions may be aborted, perhaps resulting in little progress in transaction throughput over time. From an engineering perspective, it is of critical importance to understand the factors of DBMS thrashing.

However, understanding the origin of thrashing in modern DBMSes is challenging. The challenge stems from many factors that may correlate with each other and contribute to thrashing. No structural causal model has been proposed to articulate why and when DBMSes thrash. If such a model existed, then DBAs and DBMS developers would better understand the root causes of DBMS thrashing and perhaps could predict the occurrence 
of thrashing when running their workloads on their DBMSes and could then take directed corrective actions (similarly to how errors in database design can be predicted [11] and then addressed).

\subsection{Research Questions}

In this article we address the following three research questions.

1. What factors impact the prevalence of DBMS thrashing?

2. How do the factors of DBMS thrashing relate?

3. How much variance is accounted for by each factor influencing DBMS thrashing, as well as how much variance is explained by all the proposed factors in concert?

The database community has not adequately addressed these three important questions regarding DBMS thrashing. To answer the questions, this article takes a fundamentally different approach (to be described shortly) than the analytical and simulation methods taken by a rich body of existing literature $[9,12,13,14,15,16,17,18,19,20,21,22]$.

The first question concerns what factors can contribute to DBMS thrashing. Prior research indicates that various factors, grouped into different complexity constructs (by relevance) such as i) resource complexity [7, 8, 23] defined as system resources, ii) transaction complexity [6, 9, 12, 13, 24] as transaction characteristics, and iii) processing complexity as DBMS's transaction processing capability [9, 10, 12, 19], all impact DBMS thrashing, as well as does iv) schema complexity defined as database schema, additionally considered in this article. Section 3.2 describes the complexity constructs and each of these factors in further detail.

The second question about how all the factors relate is one of the key contributions of this article. This question is addressed in Section 4. Prior research looked at the impact of one factor in isolation; this is the first time, that we are aware, that a structural causal model for DBMS thrashing has been developed and tested. In this article, we show an initial model shown in Figure 3 and then proceed with a refined model to be shown in Figure 6.

The last question concerns a quantitative approach to explaining DBMS thrashing. To answer this question, we collect empirical data on actual DBMSes. We conducted rigorous experiments by first operationalizing and then setting (for independent variables) or measuring (for dependent) variables within the context of the experiments. The measured data can then 
be used to test the relationships hypothesized from the model via a variety of statistical tools including regression [25], correlation [25], path [26], and causal mediation [27] analyses.

In the course of addressing these three questions, this article proposes a novel structural causal model that can better explicate the occurrence of thrashing in DBMSes. This model (a) helps the database community better understand the phenomenon of thrashing, (b) provides DBMS implementers and DBAs with engineering implications drawn from the model, and (c) guides researchers to identify and investigate other as-yet-unknown factors that may contribute to DBMS thrashing.

\subsection{Contributions}

This article presents the following contributions.

- Elaborates a methodological perspective that treats DBMSes as experimental subjects and uses that perspective to study DBMS thrashing.

- Proposes a novel structural causal model to explicate the origins of DBMS thrashing in transaction processing.

- Presents and tests a space of hypotheses that in concert through further investigation can refine and support (or not) the proposed model or suggest a better model.

- Extends a recent research infrastructure [28], to schedule and run large-scale experiments over multiple relational DBMSes, providing empirical data to evaluate the proposed structural causal model.

- Proposes a novel thrashing analysis protocol (TAP) that can be applied to the collected data.

- Conducts rigorous statistical analyses on the data refined by TAP.

- Suggests engineering implications to DBMS developers and DBAs, for further improving DBMSes' transaction processing.

- Guides database researchers to examine other unknown factors, contributing to DBMS thrashing, by proposing an initial tested model that can be extended and refined. 


\subsection{Organization}

The next section reviews existing related literature. Section 3 identifies several factors from the literature. We then present an initial structural causal model based on the factors and their hypothesized correlations, thereby providing a set of hypotheses to be empirically tested. Next, we describe how the factors, or variables, can be operationalized to observe their influence on thrashing and then propose a novel thrashing measurement protocol. We conduct exploratory evaluation of the initial model. Subsequently, we refine the initial model and perform confirmatory evaluation of the refined model. The model in turn provides several implications for treating workloads more efficiently. Lastly, we summarize our findings and list some remaining opportunities to augment the explanatory power by improving the amount of variance explained by the final model.

\section{Related Work}

There is a rich body of existing literature concerning load control and thrashing in transaction processing systems. Much of this work was done from the 1980's until the early 2000's [6, 9, 14, 15, 16, 17]. There have been several more recent contributions $[1,7,8,23]$ as well.

In general, there are three ways to understand any observable phenomenon (in this case, thrashing). The first way is to build and evaluate an analytical model. Tay [17] proposed an analytical model and simulation to understand, compare, and control the performance of concurrency-control algorithms. He suggested from his analytical model that data contention thrashing was due to blocking rather than to restarts, and that resource contention (competing for a transaction to finish the computation) caused thrashing to occur earlier. Möenkeberg also pointed out through his analytical model that too many active transactions in the system could incur excessive lock requests [16].

Thomasian introduced a methodology based on queuing theory to evaluate the performance of concurrency control methods in transaction processing systems [29]. Through a series of analytical models [18, 19, 20], he found out that the mean number of active transactions in the closed system increases with the MPL, reaching a maximum point before thrashing occurs, due to a snowball effect, which refers to the blocking of transactions causing further blocking of transactions.

A benefit of the analytical modeling is the conciseness of the resulting model. Once all the variables used in the model are fully understood, it is 
easy to follow the entire flow of how the model is derived. At the end, we can expect the well-summarized model in a clean mathematical form.

The second approach is simulation, used in many studies $[9,14,15,16$, 17]. One advantage lies in its flexibility [29], as a user can try various configurations in a tool. Simulation can also be used to understand complex systems. Simulation tools for extended queuing network models described in Lavenberg's book [30], may relieve a hard burden of developing simulations.

Much of the existing work relies on simulation as well as analytical models. A drawback of these analytical and simulation methodologies is that the analytic and simulation results may not hold true for actual DBMSes. It is hard to generalize their results to real DBMSes. The analytical and simulation methods can be limited in capturing complex behaviors among transactions and resources (CPU, I/O, and memory) in a current DBMS [31].

Another limitation in prior work is that the recent architectural trend of multi-core processors was not reflected. Some of the work just discussed was carried out before multi-core processors existed.

The third way is to utilize an empirical approach [32,33], measuring a real system. Recent studies $[1,7,8,23]$ used an actual DBMS. They examined transaction throughput bottlenecks that emerged on a DBMS running on a multi-core system (open source DBMSes (MySQL [1, 8], PostgreSQL [23], or their own DBMS (Shore-MT) [7]). These works aimed at improving multi-core scalability in DBMSes. They identified major bottlenecks in scalability and provided relevant engineering solutions for the DBMSes.

A drawback of this third approach is that each evaluation was conducted on one (or in a few cases, two) open-source DBMS(es). Their conclusions only apply directly to those DBMS(es). Their work could not definitively demonstrate that their results could be generalized to other (non-open source) DBMSes. As there are large differences between existing DBMSes, it is hard to say that their analysis is generalizable to other DBMSes, and even applying the tailored solution to those DBMSes may not work. As demonstrated in Figure 1, the thrashing phenomenon is observed across DBMSes. This implies that it is of critical importance to study thrashing behavior by regarding DBMSes as a general class of computational artifacts.

A recent study [34] attempts to expose and diagnose violations of atomicity, consistency, isolation, and durability (ACID) [35] - properties that modern database systems typically guarantee, under power failure. In this study the authors use a total of eight widely-used systems: three open-source embedded NoSQL (often interpreted as Not only SQL) [36, 37] systems 
(TokyoCabinet [38], LightningDB [39], and SQLite [40]), one commercial key-value store, and one open-source (MariaDB [41]) and three proprietary OLTP database servers. The approach is somewhat similar to that taken here, in that both 1) concern transactions, 2) take an empirical method using real systems, and 3) identify root causes of a phenomenon observed in database systems. However, their work focuses on understanding the causality of the ACID property violation in the presence of electrical outages, while our work aims at understanding the causality of thrashing.

None of the existing work considers correlations among factors contributing to thrashing. All previous investigations emphasize only a single factor. As more factors emerge, their interactions cannot be ignored in understanding the causality of DBMS thrashing.

In short, while there have been a good number of analytical models and simulations and empirical work to understand DBMS thrashing, the existing work has not yet broadened to address the following concerns: 1) simulation or analytical study of models rather than real DBMSes, 2) study restricted to one (or at most two) specific DBMS rather than multiple DBMSes, and 3) little consideration of relationships of factors.

In contrast, we utilize a novel, different empirical methodology, termed ergalics [42]: developing a structural causal model to better explain the DBMS thrashing phenomenon. Our empirical study 1) identifies variables affecting the thrashing phenomenon, 2) constructs a structural causal model based on the variables and their hypothesized correlations, 3) operationalizes each of the variables - determining how to measure each of the variables - and then designs experiments that set or observe each variable, 4) collects data by running the designed experiments across multiple DBMSes, and 5) tests the structural causal model via statistical techniques applied to the data. The model easily visualizes the relationships among identified factors of thrashing.

There are also some challenges using our empirical methodology. One is how to design an experimental methodology across a variety of DBMSes. It is not easy to state a consistent operationalization for a variable across very different DBMSes. The second challenge is to conduct such large-scale experiments over several DBMSes. Managing such experiments can be difficult.

\section{Exploring Potential Thrashing Factors}

There is a spectrum of granularities - depending on what is being measured and how it is measured - in building a structural causal model of DBMS 


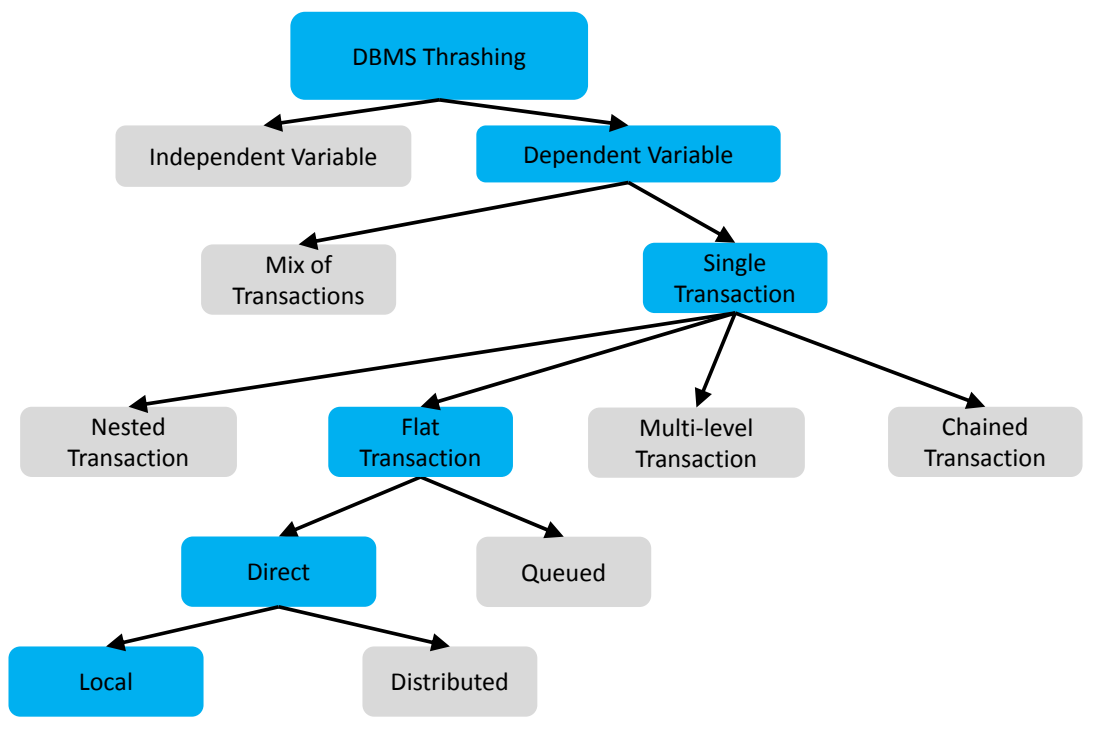

Figure 2: Taxonomy of DBMS Thrashing

thrashing. Based on this spectrum, we identify the variables of the model and collect the variables' values for testing the model.

Figure 2 presents a taxonomy for measuring DBMS thrashing. We treat DBMS thrashing as a "dependent" variable, in that it is observed, not manipulated. The variable is captured by the "maximum MPL" after which thrashing behavior emerges, called the thrashing point, discussed in Section 3.2.5.

There are several factors contributing to DBMS thrashing. These factors are treated as "independent" variables, defined as potentially impacting thrashing behavior. We introduce the independent variables in this section.

We observe the dependent variable of DBMS thrashing by operationalizing intervening (or, setting the values of) or observing (or, measure the values of) - the independent variables. In Section 5 we elaborate in detail how to operationalize them. Note that variable operationalization indicates how to set an independent variable and measure a dependent variable.

Transactions are used to observe DBMS thrashing. Transaction types can be divided into single or mixed. Single transaction type concerns a read-only or write-only transaction. A mixed transaction type involves both reads and updates within the same transaction. Here we cover only the single transaction type due to complexity of the mixed type.

In the context of Gray's categorization [43], our interest lies in a flat 
transaction that contains an arbitrary number of actions, that is, has the ACID properties [35] at the same level. A nested transaction is a tree of transactions, the sub-trees of which are either nested or flat transactions. A multi-level transaction is a special type of nested transaction that can commit ahead the results of the sub-transactions before the surrounding upper-level transaction commits. A chained transaction is the one that at commit passes its processing context to the next transaction and then implicitly starts the next transaction in one atomic operation. These other types of transactions are not covered due to complexity.

We focus on direct transactions where the terminal interacts directly with the server; in this study we do not cover queued transactions that are delivered to the server through a transactional queue interacting with clients. Lastly, we cover local transactions with no remote access. Distributed transactions are not covered in this study because of their complexity.

Before identifying the factors of thrashing, we define several terms related to transactions in the subsequent section.

\subsection{Terminology}

A transaction is represented by a single SQL statement, either a select or an update. A client is specified by a Connection instance created by a JDBC driver [44] configured for connecting to a DBMS subject, and it is implemented within a Java thread. Provided that the Connection instance has been already created, for the client to run its transaction, execute its commit, and finish its run, we 1) create a Statement object from the Connection object, then 2) invoke Statement.execute() from the Statement object by passing to that method an SQL statement representing the transaction (to be presented in Section 3.2.2), 3) call commit() to execute the commit of that transaction, 4) keep running the same transaction until a specified connection time limit (CTL), indicating how long a connection persists in the DBMS, is reached, and lastly 5) terminate the client's connection to that DBMS experiment subject.

A batch is a group of clients, each executing its own transaction. The size of a certain batch is equal to an MPL number. For the batch to run its clients' transactions, we create as many Connection objects as the size of the batch and then run each client's transaction in the batch via the aforementioned Statement.execute() in parallel. A batchset, called a "trial," is a group of batches whose size increases by steps of a certain number. The concept of 
a batchset is introduced to determine the maximum MPL, or the thrashing point. (A full view of a batchset is later given in Figure 4.)

\subsection{Relevant Variables}

Drawing from the existing literature $[6,7,8,9,10,12,13,19,23,24]$ and our understanding, we have included several independent variables (i.e., the number of logical processors, transaction size, and referenced row subset region) to be covered in detail shortly and one dependent variable (i.e., average transaction processing time) that can influence DBMS thrashing, in the initial (causal) model to be shown later in Figure 3. These variables are grouped into four constructs in the model, which are described in the following.

\subsubsection{The Resource Complexity Construct}

Resource complexity is a construct related to system resources that a DBMS utilizes. This construct includes one variable: "number of processors" (numProcs).

The Number of Processors. This is an independent variable capturing the number of logical processors (hereafter, processors) available to DBMSes. This variable addresses level of parallelism from which the DBMSes can benefit from when executing their transactions. As pointed out in Section 2, recent studies $[7,8,23]$ have paid much attention to the influence of concurrency by multi-core processors on thrashing. If we can utilize more cores, we can benefit from increased parallelism in processing. Enhanced parallelism may speed up transaction processing in DBMSes, which can service more transactions that are concurrently running. That said, the above studies actually report that as more cores are available to a DBMS, thrashing may increase because the degree of core contention becomes greater as workloads increase. The impact of multi-cores on thrashing should thus be better understood.

\subsubsection{The Transaction Complexity Construct}

Transaction complexity is a construct governing transaction properties that cause DBMS thrashing. For this construct we include the following two variables: "selectivity factor" $(S F)$ and "reference row subset region" $(R O S E)$, to be described. These two variables capture the number of rows requested by an individual transaction and the contention among transactions, respectively. 
Selectivity Factor. This variable literally indicates selectivity factor [45] of a read or write transaction. It actually captures transaction size, introduced by Thomasian [9]. Transaction size represents the number of objects requested by transactions. Transaction size can impact transaction throughput [6]. If a transaction is large, that is, if a transaction accesses many objects, many locks may be issued, and accordingly, many lock conflicts will occur when the requested objects are already held by other transactions. The lock management overhead can create substantial latency in transaction processing. Thus, transaction throughput may fall significantly. On the other hand, if a transaction is small, that is, references just a few objects, then fewer locks will be issued, fewer lock conflicts will be incurred, and thus the chance of thrashing will decrease.

Reference Row Subset Region. This variable called "referenced row subset region (ROSE)" captures the maximum region of rows (objects) that can be referenced by transactions. The variable captures effective database size in a previous study [9]. The variable is also involved with transactions' hot spots $[12,13,24]$. ROSE is specified as a percentage. The transactions can be restricted to access only a subset of rows - for instance, the first quarter $(25 \%)$ or the first half $(50 \%)$ of the rows - in a randomly-chosen table.

ROSE can affect DBMS thrashing. If ROSE is small, the degree of contention on referencing the same rows will significantly increase. Substantial locking overhead can be incurred during the transaction processing. If ROSE is large, on the other hand, contention will decrease. Many rows in the extended ROSE, however, can be referenced by transactions. Many locks on these many rows can be issued, thereby charging heavy lock management overhead and contributing to a significant drop in throughput.

\subsubsection{The Schema Complexity Construct}

The schema complexity construct concerns aspects of the relational schema that may affect DBMS thrashing. In this construct we include one variable, "presence of primary keys (PK)." This variable is identified as a factor whose presence in relations will reduce transaction processing time, thereby increasing the thrashing point.

Presence of Primary Keys. This variable captures the influence of I/O on DBMS thrashing. DBMSes can save substantial I/O in the presence of PK when treating transactions referencing PK columns in a table, by avoiding full scans through the primary index of the table. This reduced I/O can lead 
to the increase of transaction throughput, and thus, DBMS thrashing may occur later. We see that PK correlates with the thrashing point.

\subsubsection{The Processing Complexity Construct}

Processing complexity is a construct that concerns the transaction processing capability of a DBMS. In this construct we include the single variable of transaction response time.

Transaction Response Time. This is defined as the end-to-end time of completing a transaction on a DBMS.

It seems reasonable that the faster an individual transaction is processed, the higher the throughput in the end, delaying the onset of thrashing.

Certainly, the average transaction response time is lowest when one user exclusively submits transactions to the DBMS. As more active sessions are added, however, average transaction response time increases (smoothly or perhaps radically). Since having only one active session cannot realize a high throughput, typically the DBMS allows many concurrent sessions (MPL) to run their own transactions at the same time. As the number of user sessions grows, an average individual response time might be increased, but overall transaction throughput might keep rising (and perhaps falling later). Thus, we speculate that the shorter response time, the higher throughput, thereby slowing down the onset of thrashing.

However, this interaction may not hold. The response time (which also includes computation and $\mathrm{I} / \mathrm{O}$ time in performing a transaction) may rise sharply due to synchronization overhead, i.e., lock conflicts or snapshot isolation, among the concurrent transactions [9, 10, 12, 19, 22]. The overhead may eventually lead to an intolerable response time, at that point most likely resulting in a significant drop in transaction throughput, or thrashing.

In sum, transaction processing time can be an important variable to capture the impact (strength and direction) on thrashing.

\subsubsection{The Thrashing Point}

This is the dependent variable of DBMS thrashing. The variable captures a specific value of the MPL where DBMS thrashing first occurs in a given batchset. That is, it allows us to quantitatively measure the degree of thrashing observed in a DBMS. 


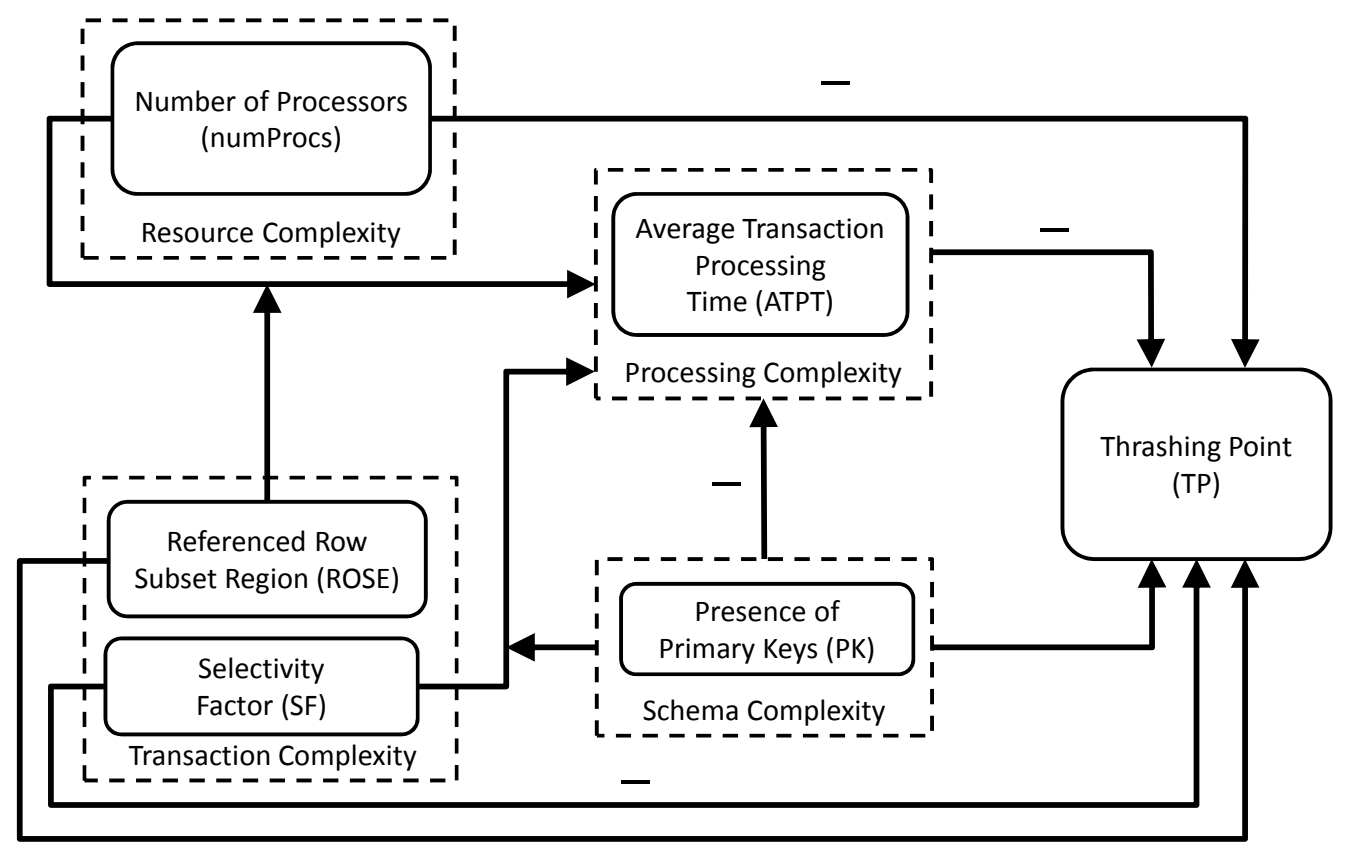

Figure 3: An Initial Model of Thrashing

\section{An Initial Model of DBMS Thrashing}

We now elaborate on an initial model of DBMS thrashing that includes the variables. We later refine this model in Section 8.

\subsection{Model Description}

Figure 3 exhibits an initial model of DBMS thrashing. This model helps us answer the second and third research questions presented in Section 1.2.

In the model we have the resource complexity construct described in Section 3.2.1. This construct abstracts system resources important to process transactions on DBMSes. The model includes this construct because of its significance on transaction throughput. In the construct we include the variable of the number of (logical) processors (numProcs), since numProcs is treated as one of the primary system resources, and it emerges as an important factor of thrashing as pointed by recent works [7, 8, 23].

Next, the model includes the transaction complexity construct described in Section 3.2.2. This construct is deeply involved in the characteristics of transactions causing throughput thrashing. The model therefore includes 
this construct. In the construct we see the variables of reference row subset region (ROSE) and selectivity factor (SF), each representing contention among transactions and transaction size influencing DBMS thrashing $[9,12$, 19, 20, 22]. These two variables concern transaction characteristics and thus are grouped into the construct together.

In the model the schema complexity construct is included. This construct includes one variable, PK, as described earlier (in Section 3.2.3). Primary keys can help DBMSes significantly reduce I/O in transaction processing. Because the PK variable concerns the relational schema, we include the variable in this construct, capturing the influence of the schema on thrashing.

In the middle of the figure is the construct of processing complexity described in Section 3.2.4. The construct captures transaction processing capability of DBMSes, impacting thrashing. The model thus has this construct. In this construct we include the variable of average transaction processing time (ATPT). Transaction-blocking by lock conflicts delays transaction processing times [9], resulting in thrashing. ATPT thus is treated as an important factor of thrashing. It depends on some of the variables in the model.

The rightmost is the dependent variable of DBMS thrashing, represented by the "thrashing point (TP)" in the model, as discussed in Section 3.2.5. An arrow from one variable (construct) to another indicates a correlation between two variables. The model includes some arrows above which a minus sign is marked, indicating a negative correlation between two variables, meaning that as a variable $X$ increases, a dependent variable $Y$ decreases.

In the model ATPT is dependent on some of the variables (numProcs and $\mathrm{PK}$ ) on its left and exerts influence on the outcome variable (TP) on its right. The relationship among these variables - numProcs, PK, ATPT, and $\mathrm{TP}$ - in the model is called mediation [25]. In other words, mediation means a relationship, such that $X$ carries two effects on $Y$, one direct from $X$ to $Y$, and the other indirect through $M$. Changes in $X$ influences changes in $M$, which in turn influences changes in $Y$. In Figure 3, for instance, $X$ corresponds to numProcs, $Y$ to TP, and $M$ to ATPT.

In addition, the model reveals that there is another relationship between numProcs and ATPT, which is conditional along with ROSE. The relationship among these variables - numProcs, ROSE, and ATPT - is called moderation [25], which means an association between two variables $X$ and $Y$ when the strength or direction depends on a third variable $M$. In Figure 3, for instance, $X$ corresponds to numProcs, $Y$ to ATPT, and $M$ to ROSE. In the moderation the effect of $X$ on $Y$ is influenced or dependent on $M$. 


\begin{tabular}{|c|c|c|c|}
\hline & $a$ & $b$ & $c$ \\
\hline I & $\begin{array}{c}\text { numProcs } / \\
\text { ATPT } \\
\text { low }\end{array}$ & $\begin{array}{c}\text { numProcs / } \\
\mathrm{TP} \\
\text { medium }\end{array}$ & $\mathrm{N} / \mathrm{A}$ \\
\hline II & $\begin{array}{c}\mathrm{SF} / \\
\mathrm{ATPT} \\
\text { high }\end{array}$ & $\begin{array}{c}\mathrm{SF} / \\
\mathrm{TP} \\
\text { high }\end{array}$ & $\begin{array}{c}\text { ROSE / } \\
\mathrm{TP} \\
\text { medium }\end{array}$ \\
\hline III & $\begin{array}{c}\mathrm{PK} / \\
\mathrm{ATPT} \\
\text { high }\end{array}$ & $\begin{array}{l}\mathrm{PK} / \\
\mathrm{TP} \\
\text { high }\end{array}$ & \\
\hline IV & $\begin{array}{l}\mathrm{ATPT} / \\
\mathrm{TP} \\
\text { medium }\end{array}$ & & \\
\hline
\end{tabular}

Table 1: Hypothesized Correlations and Levels on the Initial Model

Note particularly that the moderation concerns the mediation in the model. Such a relationship is known as moderated mediation [46]. The initial model expects that there will exist the mediation through ATPT from numProcs and from PK to TP, and in particular the mediation associated with numProcs will be conditionally influenced by or dependent on the variation in ROSE.

\subsection{Hypotheses}

Table 1 represents hypothesized correlations and levels between variables in the initial model. (A space of hypotheses drawn from the initial model is given and elaborated elsewhere [47].) Group I indicates the correlations involving the resource complexity construct with the processing complexity construct and with TP. Hypothesis I-(a) [48, 49] concerns the correlation between numProcs and ATPT. We expect the level of this correlation to be low: the enhanced parallelism by the increase of processors will improve ATPT, but since transactions are I/O-dominant and less computational, the benefit will be hidden by the substantial I/O latency. Overall, the level of the correlation will be low.

Hypothesis I-(b) $[7,8,23]$ indicates the correlation between numProcs and TP, and its strength is expected to be medium. As previously pointed out in the previous studies, processor contention is treated as one of the significant factors contributing to thrashing. Since our transactions are I/O 
dominant, however, the effect of processors on thrashing will get offset by I/O. Thus, we expect the strength to be medium.

Group II shows the correlations involving the transaction complexity construct with ATPT and TP. Hypothesis II-(a) [9, 10] indicates the correlation between SF and ATPT. We expect the strength to be high, as SF will mainly determine the amount of I/O strongly affecting ATPT.

Hypothesis II-(b) [6, 9, 10] represents the correlation between SF and TP. The level of the correlation will be high, as SF will mainly determine the amount of I/O substantially influencing transaction throughput.

Hypothesis II-(c) [9] is the correlation between ROSE and TP. When ROSE is large, row contention among transactions will get reduced. The influence of ROSE on TP will thus be little. When ROSE is small, contention for the same rows will significantly rise, thereby leading to thrashing. In that case the strength will be high. The overall level will be in the middle, or medium.

Group III indicates the correlations of PK with the processing complexity construct and TP. Hypothesis III-(a) indicates the relationship of PK with ATPT. We expect the level of this relationship to be high. As addressed before, the utilization of a primary index created by PK allows a DBMS to avoid full scans when the DBMS reads the rows referenced by transactions. The DBMS can then substantially reduce I/O time in the presence of PK. The reduced I/O time will significantly contribute to decreasing ATPT. Hypothesis III-(b) represents the correlation between PK and TP. In the presence of PK, the DBMS can increase the overall throughput because of the saved I/O time. Thus, TP can be significantly increased. Therefore, we expect the level of the correlation to be high.

Group IV shows the direct association between ATPT and TP. Hypothesis IV-(a) $[19,20]$ predicts that the increase of ATPT will decrease the number of completed transactions within unit time, resulting in a significant drop in overall throughput. In contrast, the decrease of ATPT will help DBMSes finish more transactions within unit time. Thus, the overall throughput will go up, thereby delaying the onset of thrashing. Therefore, we expect the overall strength of the correlation to be medium.

The following section discusses operationalizing each variable in the model. 


\section{Variable Operationalization}

The Number of Processors. The operationalization for this variable relies on machine specification. As described in Table 2, our individual experimental machine is configured with a quad-core processor chip supporting hyperthreading. We use four (five) values for this variable: one processor specified by one core with hyper-threading disabled, two processors by one core with hyper-threading enabled, four processors by two cores with hyper-threading enabled, (six processors by three cores with hyper-threading enabled,) and eight processors by four cores with hyper-threading enabled. In Linux, we can alter numProcs by setting a specific number to "maxcpus" available in /boot/grub/grub.conf. For Windows, numProcs can be altered by our proficient lab staff via a BIOS option.

Selectivity Factor. The way we operationalize the variable is to vary the number of rows accessed by each transaction. As mentioned before, the type of transaction is read-only or write-only. For simplicity, we use a single SQL statement within each transaction, to be exemplified shortly.

A read-only transaction can be specified by a SELECT statement. For a read-only transaction, stating $1 \%$ as the maximum SF, we vary the SF from $0.01 \%$ up to $1 \%$ by factors of 10 . If $\mathrm{SF}$ is set to $1 \%$, then we generate a transaction reading $1 \%$ of consecutive tuples in a randomly-chosen table. The maximum of $1 \%$ is determined to be that needed to obtain a reasonable TPS value at an MPL. (It is not strictly necessary to cap the maximum SF at $1 \%$, though an SF greater than $1 \%$ may not be prevalent in real-world applications.)

A write-only transaction can be specified by an UPDATE statement. We don't use INSERT and DELETE for the write-only transaction, because the two statements change the cardinality of a table, which introduces other effects, thereby confounding the experiment. In contrast, UPDATE preserves the same semantics for operationalizing the write-only (hereafter, update-only) transaction, because it avoids a change in cardinality. The influence of insert/deletes on thrashing may be investigated in the future.

Unlike a read-only transaction where the maximum $\mathrm{SF}$ is $1 \%$, for a update-only transaction we vary the SF linearly. Instead we try 1\%, 0.75\%, $0.50 \%$, and $0.25 \%$. So if SF is set to $1 \%$ we generate transactions updating $1 \%$ of tuples in a randomly-chosen table. The reason for taking different scales for each type of transaction is that the linear variation of update-only 
SF is sufficient to see thrashing, considering that an update-only transaction incurs exclusive locks while a read-only transaction incurs shared locks.

The SQL statements associated with the read-only and update-only transaction will be shown shortly, after we discuss the following variable.

Reference Row Subset Region. We operationalize this variable in the following manner. For each transaction in a batch we first randomly choose a table from our database schema and define the entire table rows as $100 \%$. We can then decrease the proportion of the rows to $75 \%, 50 \%$, and $25 \%$ from $100 \%$ for ROSE. For example, if ROSE is set to $50 \%$, then a transaction can be restricted to reference only the first half rows in the randomly-chosen table. If ROSE is set to $100 \%$, then all the rows in the table can be referenced by the transaction. The value of ROSE will determine the range of rows that a transaction can reach, and it will be used for specifying and restricting the transaction.

Given a combination of values of $\mathrm{SF}(s)$ and $\operatorname{ROSE}(r)$ as described above, a read-only or update-only transaction to be generated will look like the corresponding one as follows:

\begin{tabular}{l|l} 
Read-only trans. & Update-only trans. \\
\hline SELECT column_name & UPDATE table_name \\
FROM table_name & SET column_name $=v$ \\
WHERE $i d 1>=a$ and $i d 1<b$ & WHERE $i d 1>=a$ and $i d 1<b$
\end{tabular}

where table_name is a randomly-chosen table in the schema, $a$ is an integer value randomly chosen in the interval of $[0,(c \times r)-(c \times s)], b$ is $a+c \times s, c$ is the cardinality of the table $(1 \mathrm{M}$ or $30 \mathrm{~K}), v$ is an integer value or a character string randomly generated along with a chosen column (column_name), and id1 is a sequential row ID.

For simplicity we use transactions on a single table; more complicated transactions over multiples tables are left as future work.

Presence of Primary Keys. This variable is a dichotomous variable (that is, primary keys are either present or absent). We can operationalize PK by telling a DBMS whether or not to treat id1 as the primary key column of a table when populating the table.

Batchset Generation and Execution. As visualized in Figure 4, a batchset (termed in Section 3.1) contains a set of ten batches, each successively increasing by 100. Thus, the biggest batch in the batchset consists of 1,000 


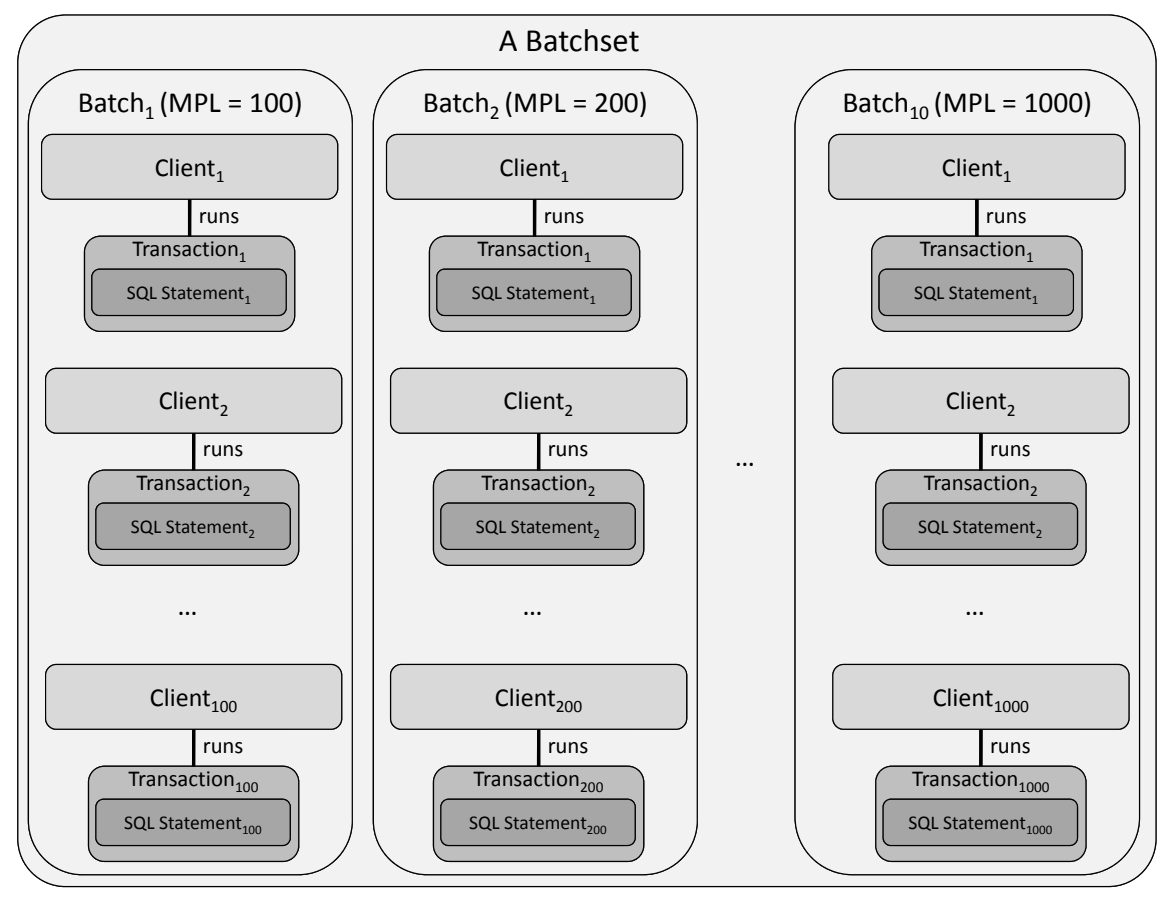

Figure 4: Batchset Visualization

clients. Each client in a batch has its respective transaction generated in the way mentioned in the paragraph of ROSE. Transactions used in our experiment as shown in Figure 1 use a simple database schema. The schema is composed of two tables, each consisting of seven columns, five of which are non-null integers, and the other two are variable-sized character columns. A value for the first integer column, called id1, is sequentially generated (and used for operationalizing SF). Each tuple is 200 bytes long, consisting of the total 20 bytes from the integer type columns and the total 180 bytes from the two character columns. Each table is populated with $1 \mathrm{M}$ (or $30 \mathrm{~K}$ ) tuples having column values randomly-generated except the first column (for the $\mathrm{DBMS}^{3}$ running on Windows).

A prepared batchset is presented to a DBMS subject. At each value of MPL (equivalent to a batch) in that batchset we run all the transactions of the clients in that batch until a CTL (connection time limit) value - two

\footnotetext{
${ }^{3}$ The above $30 \mathrm{~K}$ cardinality was chosen only for this DBMS, as populating tables with $1 \mathrm{M}$ on that DBMS was not successful due to unknown system errors.
} 
minutes - is reached, as described above in Section 3.1, and we make the same batch run multiple times for repeatability. In each batch run we 1) measure via JDBC and record the elapsed times of the transactions executed on that DBMS and 2) count the number of completed transactions. These two measured data are individually used to later measure the values of dependent variables introduced in Sections 3.2.4 and 3.2.5, whose operationalization is discussed in the following.

Transaction Response Time. This variable is captured in our model by average transaction processing time (ATPT). We cannot directly control the variable, but we can measure it. To operationalize (measure) this variable, as mentioned above we complete a run of a given batchset. We then average the recorded elapsed times for all the transactions executed in the batchset. The averaged elapsed time is then the ATPT associated with the batchset. This method can be applied to any DBMS. It is hard to directly observe what locking schemes on objects (pages, tables, tuples, and columns) are employed by different DBMSes, but the calculated ATPT seems to not only reflect the impact of those schemes on TP but also captures different characteristics among the DBMSes with respect to code and performance.

Thrashing Point. To operationalize (observe) the thrashing point, for a completed run of a given batchset we 1) compute the average transactions per second (TPS) for a batch, based on the recorded data of the number of executed transactions for multiple runs of that same batch, 4) calculate the average TPS for each of the remaining batches in the batchset in the same way, and finally 5) derive the thrashing point (TP) for the batchset based on the following Equation 1:

$$
\mathrm{TP}= \begin{cases}\left|B_{i}\right| & \text { if } T P S_{i}>1.2 \times T P S_{i+1}, \text { and } \forall j>i+1, T P S_{i}>T P S_{j} \\ \infty & \text { otherwise }\end{cases}
$$

where $i>0, B_{i}$ is the $i$-th batch in a given batchset, and $T P S_{i}$ is the measured TPS for $B_{i}$.

A specific TP is equal to the size of a batch (that is, a particular MPL), at which the measured TPS value is more than $20 \%$ of the TPS measured at the subsequent batch, and it is greater than the rest of the values of TPS measured at all the subsequent batches in the same batchset. As illustrated in Figure 1(a), for instance, the TP of DBMS $X$ is an MPL of $800\left(\left|B_{8}\right|\right)$, as the TPS measured at an MPL of $900\left(\left|B_{9}\right|\right)$ was beyond $20 \%$, lower compared 
with that of the previous MPL of 800. Also, the TPS at the TP was higher than the TPS at the last MPL of 1,000. In the figure that each point (or MPL), in steps of 100, corresponds the size of each batch in the batchset used in that experiment. Once thrashing begins, the observed thrashing should continue until the last MPL in a trial.

The calculated TP quantitatively represents how many MPLs can be tolerated by a DBMS for the batchset. The $20 \%$ threshold was determined based on our observation, such that once a measured TPS dropped by $20 \%$ or more than a previous TPS (at the TP), there was no case that subsequent TPSes were bigger than the TP's TPS.

In the following we describe a methodology for measuring DBMS thrashing as TP, to collect empirical data, necessary to test the aforementioned hypotheses in Section 4.2 .

\section{Thrashing Metrology}

Metrology is the science of weights and measures or of measurement [50]. In this section we discuss the overall thrashing metrology for measuring DBMS thrashing based on the variables described in the previous section. The metrology includes a thrashing observance scenario, experiment infrastructure, and data analysis protocol.

Before elaborating on the scenario, we introduce the configurations of our system used for the experiments in the next.

\subsection{Experiment Configuration}

Table 2 summarizes the system configuration used in the experiments in our study. Each experimental machine is of identical hardware $(\mathrm{H} / \mathrm{W})$ : i) a quad-core processor chip supporting hyper-threading, ii) 8GB RAM, and iii) 1TB SATA HDD with 7200 RPM. Each machine runs Red Hat Enterprise Linux (RHEL) 6.4 with a kernel of 2.6.32, except one running Windows Server 2008 dedicated for one of the DBMSes. Each machine exclusively runs one of the five DBMSes.

This machine configuration represents a single value for these controlled independent variables (whereas in Section 5 we listed other independent variables, which are each given several values). While we do not vary the system configuration, we feel that other configurations (thus, other values for these independent variables) could exhibit similar predicted behavior. Thus our causal model predicts certain interactions and certain behaviors (values of 


\begin{tabular}{|c|c|c|c|}
\hline \multicolumn{4}{|c|}{ System Specification } \\
\hline \multirow[t]{3}{*}{$\mathrm{H} / \mathrm{W}$} & $\mathrm{CPU}$ & \multicolumn{2}{|c|}{$\begin{array}{l}\text { Intel Core } i 7-870 \text { Lynnfield } 2.93 \mathrm{GHz} \text { LGA } 1156 \\
\text { 95W Quad-Core Processor (in one socket) }\end{array}$} \\
\hline & RAM & \multicolumn{2}{|c|}{$\begin{array}{l}\text { Kingston ValueRAM 8GB (4GB }(2 \times 2 \text { C } \times \text { 2G) } \\
\text { 240-Pin DDR3 SDRAM DDR3 } 1333 \text { (PC310600) } \\
\text { Dual Channel) }\end{array}$} \\
\hline & HDD & \multicolumn{2}{|c|}{$\begin{array}{l}\text { Western Digital Caviar Black WD1001FALS 1TB } \\
7200 \text { RPM SATA } 3.0 \mathrm{~Gb} / \mathrm{s} 3.5 \text { " Internal Hard } \\
\text { Drive }\end{array}$} \\
\hline \multirow{5}{*}{$\mathrm{S} / \mathrm{W}$} & \multirow[t]{2}{*}{ OS } & $\begin{array}{l}\text { Linux } \\
\end{array}$ & $\begin{array}{l}\text { RHEL release } 6.4 \text { Santiago (kernel } \\
2.6 .32)\end{array}$ \\
\hline & & Windows & Windows Server 2008 R2 Enterprise 6.1 \\
\hline & \multirow{3}{*}{ DBMS } & \multicolumn{2}{|c|}{ MySQL 5.5.8 } \\
\hline & & \multicolumn{2}{|c|}{ PostgreSQL 8.4.9 } \\
\hline & & \multicolumn{2}{|c|}{ three proprietary DBMSes $\mathrm{X}, \mathrm{Y}$, and $\mathrm{Z}$} \\
\hline
\end{tabular}

Table 2: Environment Setup

the dependent variables) as impacted by the specific settings of the independent variables. We mention in passing that we assume the configuration DBMSes i) support a concurrency control model of locking or snapshot isolation, ii) rely on a filesystem (rather than direct I/O), and iii) are able to support up to 1,000 connections (threads).

\subsection{The Thrashing Observance Scenario}

Figure 5 depicts our thrashing measurement scenario, the structure of our experiments running a hierarchy of eight levels, ending at a particular execution of a particular batch (at an MPL) of a particular batchset for the underlying table(s), as part of a particular experiment run started on a stated date and time on a designated machine using a specified release of a specific DBMS, in the context of a specified experiment setup (stating batchsets, the characteristics of the data, and various other parameters), of a selected experiment scenario.

For each batch execution (during the two-minute CTL), the number of completed transactions is counted to derive transactions per second (TPS). We make five batch executions for each batch. For each batch TPS gets averaged among the batch executions. 


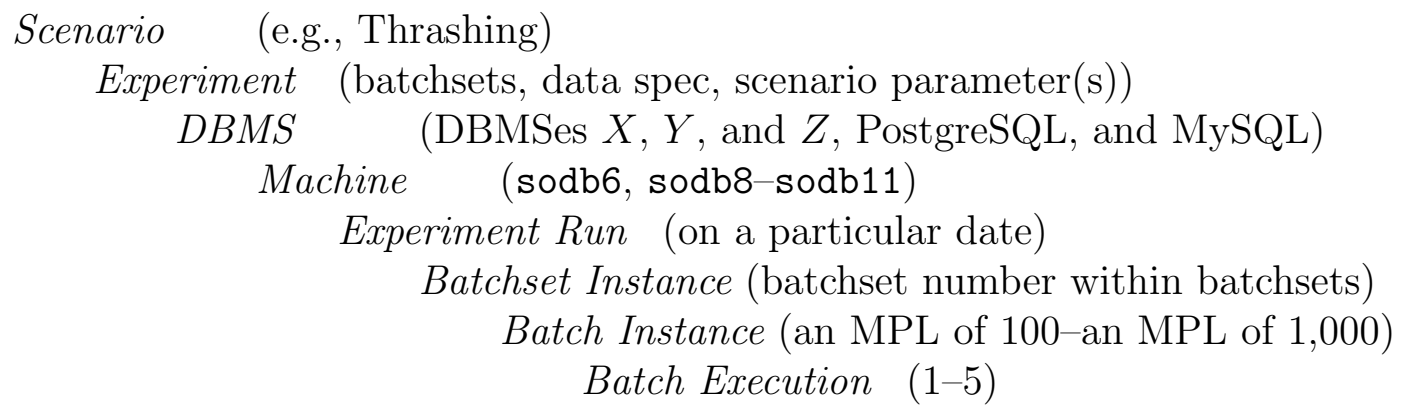

Figure 5: Hierarchy of Batch Executions

As an example of this hierarchy, each point in Figure 1 corresponds to the averaged TPS at each MPL. For the data in Figure 1, we utilized the Thrashing Scenario, an Experiment specifying a nineteenth set (BS19) of 28 batchsets, specifying data with a cardinality of $1 \mathrm{M}(30 \mathrm{~K})$ rows, and specifying the scenario parameters of 1) five executions per batch, 2) one core with hyper-threading disabled, and 3) declared primary keys. We ran this batchset on DBMSes $X, Y, Z$, MySQL, and PostgreSQL on the sodb9, sodb8, sodb6, sodb11, and sodb10 machines from experiment runs for batchset number 19, respectively, thus identifying a particular batchset instance, and examining all ten batches (MPLs).

The Thrashing scenario is a plug-in that we added to a laboratory information system, called AZDBLAB [28].

\subsection{AZDBLAB Infrastructure}

AZDBLAB is a DBMS-oriented research infrastructure, supporting largescale scientific studies across different DBMSes [28]. It has been developed for more than seven years by many people's contribution. AZDBLAB is operated on a hardware lab of dedicated machines: one each for each DBMS subject and one to host the central DBMS server to store data collected from the DBMS subjects. Currently, a total of seven relational DBMSes are supported, both open source and proprietary. The infrastructure has been sufficiently robust to collect empirical (query execution) data on the Linux and Windows operating systems, totalling over 9100 hours (more than one year, $24 \times 7$ ) of cumulative time. 


\subsection{Running Experiments}

Leveraging AZDBLAB, we made tens of experimental runs of the Thrashing scenario. To run the scenario, we used five relational DBMS subjects available in AZDBLAB, as shown in Figure 1. (The remaining two DBMSes could not be used because of some technical issues that emerged on those two while running the scenario.)

While running the experiments AZDBLAB collected each batchset's transaction elapsed time and TPS data measured on the DBMS subjects.

Our experience studying varying query time $[51,52]$ and query suboptimality [53] is that AZDBLAB facilitated developing and running the thrashing scenario with multiple configurations of the experiments. (That said, this does not mean that AZDBLAB is a requirement for this thrashing study.) We plan to release AZDBLAB sometime in the next year after enhancing the system.

Before proceeding with the evaluation of the model using the measured data, we asked two related questions: How could we make sure that the data were clean enough to proceed with the evaluation of the model? And is there a way of assessing the validity of the acquired data? To address this underlying concern, we designed a sophisticated data analysis protocol.

\subsection{Thrashing Analysis Protocol}

The protocol, termed Thrashing Analysis Protocol (TAP), performs a suite of sanity checks on the measured data, drops data failing to pass the checks, and then computes the thrashing point for each batchset in the retained data. TAP consists of the four steps.

In Step 1 TAP conducts eight sanity checks partitioned into three classes. The first class is the experiment-wide cases for which not a single violation should occur in a run, consisting of four sanity checks. (1) The number of missing batches indicates how many batch instances (BIs) that were supposed to have been executed with a batchset (10), but were not, for whatever reason. (2) The inconsistent processor configuration violation occurs when the number of processors specified in a given experiment specification is inconsistent with the current processor configuration on an experiment machine. (3) The number of missed BE violations represents how many BIs did not have the specified number of repetitions required. (4) The other DBMS process violation identifies how many BSIs were run together with other DBMS processes. We enforce that when a BSI gets run on a chosen DBMS, all the other DBMSes' processes should be deactivated. 
The second class of sanity checks concerns batch executions (BEs). Each of these three sanity checks could encounter only a few isolated violations, but we expect the violation percentage to be low. (5) The zero TPS violation catches BEs where TPS was zero at the starting MPL in their BSIs. (6) The connection time limit violation identifies the BEs that violated the specified CTL. (7) The abnormal ATPT violation indicates how many BEs revealed abnormally high or low ATPT.

The final class involves one check over a BSI. (8) The transient thrashing violation examines how many BSIs experienced transient thrashing, which indicates that the TPS measured at the thrashing point (TP) is lower than any of the TPSes measured at the MPLs bigger than the TP. Once the thrashing phase begins, it will be rare to observe that the throughput rises back up at greater MPLs.

In Step 2 TAP drops BEs that exhibit specific problems throughout Step 1. We take the union of BEs caught by the second class of sanity checks $((5),(6)$, and $(7))$, and remove those BEs from further consideration.

In Step 3 TAP looks at the retained BEs for each BSI and then determines if these BEs in concert exhibit specific problems. If so, we drop the entire BSI (having its own BEs). Step 3 consists of the three sub-steps. Step 3-(i) drops BSIs that do not have all the MPLs in the corresponding BSIs. Recall that each batchset has ten batches. If there is any BI dropped in the same BSI, we drop that BSI. Step 3-(ii) drops raw BSIs revealing transient thrashing, as specified in (9). Step 3-(iii) drops retained BSIs showing transient thrashing.

In Step 4 TAP calculates the TP for each of the retained BSIs along with Equation 1. After finishing Step 4, we proceed with conducting (exploratory or confirmatory) evaluation on the model with the measured data: the retained BSIs and their corresponding TPs.

Now we discuss exploratory evaluation of the initial model.

\section{Exploratory Evaluation of the Initial Model}

In this section we summarize the experimental data used for the exploratory evaluation and present the evaluation results on the initial model.

\subsection{Descriptive Statistics}

For the exploratory evaluation we used the aforementioned five relational DBMSes. We operationalized four different numProcs values and two values (that is, presence and absence) for PK. As exhibited in Table 3, we completed 


\begin{tabular}{|l|c|c|}
\hline & Exploratory & Confirmatory \\
\hline Number of Experiment Runs & 40 & 50 \\
\hline Number of BatchSet Instances (BSIs) & 1,120 & 1,400 \\
\hline Number of Batch Instances & 11,200 & 14,000 \\
\hline Number of Batch-instance Executions (BEs) & 33,600 & 70,000 \\
\hline Total Hours & 3,127 & 5,170 \\
\hline
\end{tabular}

Table 3: Desc. Stat. for the Exploratory and Confirmatory Exp. Runs

40 experimental runs, each taking a few days to a week on a single machine (with the disk drive humming the entire time), totaling 3,127 hours (about 4 months) of cumulative time.

Considering that each of the 40 completed runs contained 28 batchsets (operationalized by a combination of four ROSE values and seven SF values), we had a total of $28 \times 40=1,120$ BSIs. As each BSI had ten batches, we had $1,120 \times 10=11,200 \mathrm{BIs}$. As each batch was executed three times, we had 11,200 BIs $\times 3=33,600$ BEs. In short, our exploratory study concerns the 40 completed runs, involving 33,600 BEs.

TAP was applied to the 33,600 BEs. In Step 1 we identified all the BEs revealing problems. We then dropped in concert 2,507 BEs (about 7.46\%) in Step 2. In Step 3 we subsequently dropped in concert 109 BSIs (about 9.8\%) (from 1,112 BSIs calculated based on the BEs retained after Step 2). In effect, a total of 1,003 BSIs survived throughout Steps 2-3. For the retained 1,003 BSIs we calculated the TPs based on Equation 1 in Step 4. As a result, we detected a total of 487 thrashing BSIs from the five DBMSes.

Several conclusions from the thrashing BSIs can be drawn. First, every DBMS exhibited thrashing. The number of thrashing BSIs differed by about a factor of three among the five DBMSes. The DBMS thrashing phenomenon thus must be a fundamental aspect of either the algorithm (concurrency control), the creator of the algorithm (human information processing), or system operation context (environment). Note that our model covers all of these effects. About half of the BSIs (487 out of 1,003) exhibited thrashing somewhere in the range of varying MPLs in the exploratory experiment; in particular, there were of the thrashing 487 BSIs for which the DBMSes revealed "early" thrashing under MPL 300.

The thrashing 487 samples consisted of a group of 188 read batchsets (hereafter, read group) and a group of 299 update batchsets (hereafter, update 
group). Our evaluation was performed separately on these two groups.

\subsection{Exploratory Evaluation Results}

In this section we discuss the statistical analysis results on the initial model in Figure 3.

\subsubsection{Correlational Analysis}

We performed correlational analysis using cor.test() of R [54]. We tested hypothesized correlations and levels exhibited in Table 1.

For the read group, I-(a), III-(a), III-(b), and IV-(a) were supported while II-(a), II-(b), and II-(c) were not supported. I-(b) was not supported, because the actual direction of its correlation was positive while the hypothesized direction was negative as depicted in Figure 3. In the update group I-(a), I-(b), and IV-(a) were supported whereas II-(a), II-(b), and II-(c), and III(a), III-(b) were not supported. It seemed that the variables of SF and ROSE had some unknown challenges in empirically observing correlations with ATPT and TP.

\subsubsection{Causal Mediation Analysis}

We performed causal mediation analysis [27] via mediate() in the mediation package in $\mathrm{R}$. We tested the mediation through ATPT to TP, moderated by ROSE and PK. In both groups the moderated mediation was not significant, perhaps because of ROSE and SF that were not significant. However, the mediation through ATPT to TP from numProcs was significant, as was that from PK in the read group and from numProcs in the update group.

\subsubsection{Regression Analysis}

The performance of the model as a whole was evaluated via the value of $R$-squared $\left(\mathrm{R}^{2}\right)$ [25], the ratio of explained variance to sample variance of a dependent variable of the model. The computed $\mathrm{R}^{2}$ tells us how well the model can fit the measured data. If the value of $\mathrm{R}^{2}$ is close to $1(100 \%$ variance), all contributing factors are identified. If the $\mathrm{R}^{2}$ is close to zero ( $0 \%$ variance), the model is not useful, as the factors included in the model cannot explain much of the variance.

Our regression analysis was conducted using multi-linear regression, a well-known statistical tool for modeling the relationship between a continuous dependent variable and multiple independent (explanatory) variables that are 
continuous or dichotomous [25]. MLR was a perfect fit for the initial model, satisfying the variable condition for the application of MLR. (Testing the assumptions of MLR is discussed in Section 9.2.)

Using MLR, we first performed regression on TP. We used $\operatorname{lm}()$ of $R$ and examined the overall fit of the model on each group. The amount of variance explained $\left(R^{2}\right)$ for TP was 11\% (14\%) for the read group (update group). We also performed regressions on ATPT that has dependency on the variables in the model. The initial model explained $12 \%$ (11\%) of the variance of ATPT for the read (update) group.

\subsubsection{Path Analysis}

We performed the path analysis on the initial model, to determine which paths were statistically significant, using regression coefficients provided by the outcome of $\operatorname{lm}()$.

In the read group the significant paths were (a) from numProcs to ATPT, (b) from PK to ATPT, (c) from numProcs to TP, (d) from ATPT to TP, and (e) from PK to TP in Figure 3, and their weights were (a): $-0.19,(\mathrm{~b})$ : $-0.30,(\mathrm{c}): 0.11,(\mathrm{~d})-0.17$, and (e): 0.25 , respectively.

On the other hand, there were not significant paths: (i) from ROSE to $\mathrm{TP}$, (ii) from SF to TP, and (iii) from SF to ATPT, in addition to (iv) from numProcs to ATPT, moderated by ROSE, and (v) from SF to ATPT, moderated by PK. We thought we understood these paths, and thus we included them in the initial model. However, it seemed that the operationalization of ROSE and SF unexpectedly incurred confounding behavior. In future work, other ways of operationalizing these variables can be further explored, and we discuss possible improvements in Section 7.3.

To continue our model exploration, we performed a regression analysis on the refined model, removing the confounding paths. Consequently, all the retained paths in the refined model were significant. Their weights were (a): -0.12 , (b): $-0.29,(\mathrm{c}): 0.13$, (d) -0.17 , and (e): 0.22 , respectively. There was little difference between the corresponding weights of the initial and refined models. In other words, there was little discrepancy between the initial and refined models. This implies that the refined model did not fit the data any worse than the initial model including the eliminated paths.

In the update group the significant paths were (f) from numProcs to ATPT, (g) from numProcs to TP, and (h) from ATPT to TP in Figure 3, and their weights were $(\mathrm{f}):-0.29,(\mathrm{~g}):-0.45,(\mathrm{~h}):-0.11$, respectively.

The above insignificant paths in the read group also resulted in being not 
significant in the update group, plus the paths involving PK. Again, we included all these paths in the initial model for the update group, as we thought we understood them, which was not true. The better operationalization of PK as well as ROSE and SF need be investigated in the future.

As did in the read group, we performed a regression analysis on the refined model, eliminating the paths that were not significant. As a result, the refined model yielded no insignificant paths. The respective weights of (f), (g), and (h) were $-.30,-0.43$, and -0.10 . Both the initial and refined models did not reveal statistical discrepancy. Hence, the refined model did not fit the data more poorly than did the initial model on the update group.

\subsection{Lessons}

We have learned several things from the preliminary study on the initial model. First, some variables were not statistically significant. Specifically, the associations of ROSE and SF with ATPT and TP (thrashing point) were not significant for the read and update groups. In addition, the correlations involving PK were not significant in the update group. There might be a general lack of understanding of those variables or perhaps their operationalization.

Here are some possible suggestions to better operationalize the variables. Concerning ROSE one way would be to have each transaction in a batch reference a contiguous chunk of table rows in sequence within a specified region (by a ROSE value). If a transaction reaches the last chunk of rows in the region, then the next transaction could access the first chunk of rows at the beginning of the region. If so, each row in that region would be referenced with equal probability by the transactions. Another would be to have every transaction access the same number of discrete rows (using 'IN' predicate) with even access probability. For SF, it would be possible to consider extending the value range from the minimum cardinality (or a single row) to the maximum cardinality (or $1 \mathrm{M}$ rows) of the table.

Also, it would be worth exploring the existence of other variables perturbing the associations involving ROSE, SF, and PK (only in the update group).

Second, the read and update groups showed differences in the observed correlations. 1) The direction of a correlation between the same corresponding variables was not consistent between the read and update groups. The correlation of numProcs with $\mathrm{TP}$ was positive in the read group whereas it was negative in the update group. 2) PK was not significant in the update 
group while it was significant in the read group. 3) The amount of variance explained by the model for the read group was different than that of the update group.

Third, we observed across the DBMSes different thrashing behaviors between the read and update groups. Specifically, for DBMS $X$ the average TP of the update (read) group was about 614 (870), and for DBMS $Y$, it was about 266 (462) in our exploratory experiments. The other DBMSes also revealed a similar tendency: the overall thrashing on the update group occurred earlier, perhaps because of heavier locking overhead.

For these reasons, we concluded that our model should be divided up into two parts, explicating the respective thrashing origins for the read and update groups, resulting in the refined model to be discussed now.

\section{A Refined Model of DBMS Thrashing}

In this section we give a refined model of DBMS thrashing and present and discuss in detail a space of hypotheses related to the refined model. The refined model is depicted in Figure 6. The specific values above arrows result from our path analysis to be discussed in Section 9.3.4. We elaborate on the values in that section.

\subsection{A Refined Model for the Read Group}

As seen in Figure 6(a), the variables of ROSE and SF and their associated associations were removed because we do not adequately understand how they interact with other variables of interest. The direction of the correlation between numProcs and TP was changed from negative to positive because we observed that the benefit of utilizing more processors for the read group dominated the expected contention among the processors, we feel due to much less locking overhead. So we now expect the direction to be positive.

Let's examine the set of hypotheses drawn from the refined model in Figure 6. Such relationships are specific associations between the constructs (or, their variables), as hypothesized by this predictive causal model.

One factor of the read model is the resource complexity construct. We hypothesize that this construct affects DBMS thrashing both directly, as depicted by the top line, and indirectly, via ATPT.

Consider numProcs first. Multicores present a great opportunity to DBMSes $[8,23]$. Multi-core processors enable DBMSes to process more transactions than a single processor does, due to increased parallelism. The pre- 


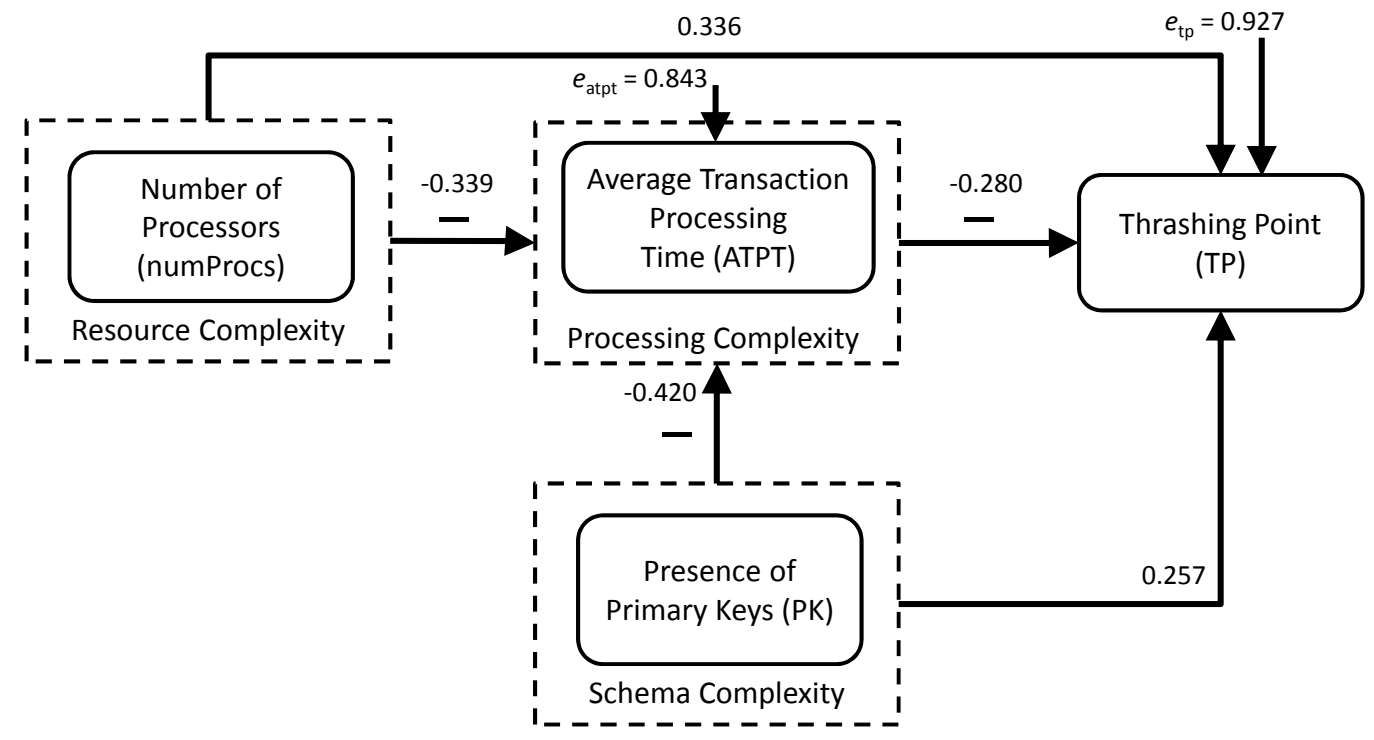

(a) The Model of the Read Group (Read Path Model)

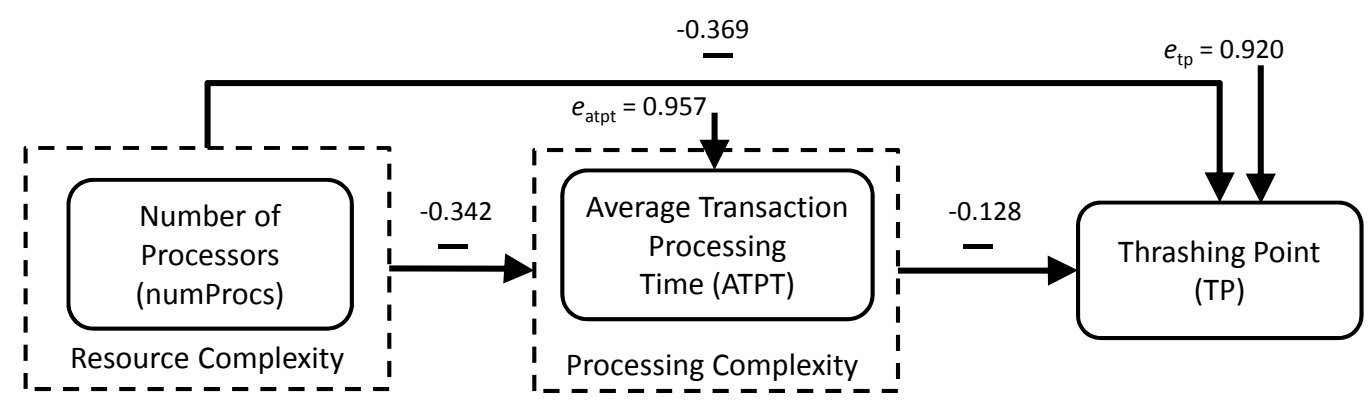

(b) The Model of the Read Group (Update Path Model)

Figure 6: A Refined Model of DBMS Thrashing (Path Diagrams on the Batchset Groups in the Confirmatory Experiment) 
vious studies [8, 23], however, reported that DBMSes experienced thrashing by multicores because of core contention, but their studies were conducted on one specific DBMS as mentioned in Section 2. In general, transaction processing in most DBMSes can be sped up by using multi-core processors. We thus hypothesize the following.

Hypothesis 1: As the number of processors increases, the thrashing point will increase.

As mentioned before, the increased parallelism using more processors can speed up transaction processing. Although overall throughput could fall off due to contention among the processors, we anticipate that as far as individual transaction response time is concerned, it will be shorter when more processors are presented.

Hypothesis 2: As the number of processors increases, ATPT will decrease.

Concerning processing complexity, ATPT directly influences DBMS thrashing. Specifically, as transactions' response time gets shorter, the overall throughput can rise. On the contrary, as the response time gets longer, the throughput may significantly fall at a certain point, resulting in thrashing.

Hypothesis 3: As ATPT increases, the thrashing point will decrease (i.e., thrashing will occur earlier).

We hypothesize that in the presence of PK, TP will increase. PK allow DBMSes to avoid doing full scans on the tables referenced by transactions. When PK exists, a DBMS can thus speed up processing transactions accessing PK columns. As a result, the overall transaction throughput will increase, leading to an increase of TP as well.

Also, we hypothesize that ATPT will decrease if PK exists. Considering that $\mathrm{I} / \mathrm{O}$ is one of the significant components in transaction processing, DBMSes can significantly benefit from a primary index automatically created when the PKs are declared. The I/O saved through a primary index will suppress a significant rise in processing delay incurred when transactions simultaneously read or update rows. Furthermore, for a given SF, ATPT will be reduced in the presence of PK. That is, the slope between SF and ATPT will be lower when PK is present.

Hypothesis 4: In the presence of primary keys the thrashing point will increase. 
Hypothesis 5: In the presence of primary keys, ATPT will decrease.

\subsection{A Refined Model for the Update Group}

ROSE and SF were eliminated in both portions of the model. PK was also removed, with its associated correlations. In comparison with Figure 6(a), the direction of the association between numProcs and TP was retained because of the supported hypothesis on the association.

Now let's discuss the hypotheses drawn from the update model. In contrast to the read model, we focus on a drawback in taking advantage of multicore processors in update transaction processing. Enabling more processors could incur serious competition among the processors in utilizing shared resources available within the DBMS internals. Increased core contention can hurt transaction throughput, or slow down transaction processing.

Hypothesis 1: As the number of processors increases, the thrashing point will decrease.

Concerning the processing complexity construct, we expect that the more processors, the faster ATPT in the update group, because of the same reason described in the read model.

Hypothesis 2: As the number of processors increases, ATPT will decrease.

For the same reason described in the read model, we expect that the longer ATPT, the shorter TP.

Hypothesis 3: As ATPT increases, the thrashing point will decrease (i.e., thrashing will occur earlier).

\subsection{The Revised Hypothesized Correlations and Levels}

Table 4 exhibits the eight hypothesized correlations on the refined model. The corresponding levels, separated by '/' for the read/update groups, are shown in parentheses. Note that compared to Table 1, group II no longer exists because the variables of ROSE and SF and relevant correlations are removed in the refined model. In the read group every cell is as in Table 1, except the levels that get decreased to medium from high or increased to medium from low. This is because of our observation that these correlations were weaker or stronger than we thought in the initial model.

In the update group we do not have group III because PK is not present in the refined model. The levels in group III are thus denoted as 'N/A.' Also, the level of Hypothesis IV-(a) is decreased to low from medium compared to 


\begin{tabular}{c|c|c|} 
& $a$ & $b$ \\
\hline \multirow{3}{*}{ I } & numProcs / & numProcs / \\
& ATPT & TP \\
& $($ low / medium $)$ & $($ low / low $)$ \\
\hline \multirow{2}{*}{ III } & $\mathrm{PK} \mathrm{/}$ & $\mathrm{PK} /$ \\
& ATPT & $\mathrm{TP}$ \\
& $($ medium / N/A) & (medium / N/A) \\
\hline \multirow{2}{*}{ IV } & $\mathrm{ATPT} /$ & \\
& $\mathrm{TP}$ & \\
& (medium / low $)$ &
\end{tabular}

Table 4: Revised Hypothesized Corr. and Lev. on the Read/Update Groups

Table 1, as we saw that this correlation was weaker than we thought in the initial model.

\section{Confirmatory Evaluation of the Refined Model}

In this section we provide descriptive statistics on the experimental data used for the confirmatory evaluation and present statistical analysis results on the refined model.

\subsection{Descriptive Statistics}

For the confirmatory evaluation, we used the same five relational DBMSes and kept the same operationalization except increasing one more value for numProcs. As previously provided in Table 3, we performed 50 experiment runs, totaling 5,170 hours (about 7 months) of cumulative time. Note that this data was completely separate from that used in the exploratory evaluation discussed above.

We had a total of 28 (batchsets) $\times 50$ (runs) $=1,400$ BSIs. As each BSI had ten batches, we had 1,400 $\times 10=14,000$ BIs. As each batch was executed five times, we had 14,000 BIs $\times 5=70,000$ BEs. In short, our confirmatory study concerns the 50 completed runs, involving 70,000 BEs, more than twice as many BEs as the exploratory study had.

As was done in the exploratory study, TAP was applied to the 70,000 BEs. Tables 5, 6, and 7 exhibit the step-wise TAP sanity check results. Table 5 shows no violation in the experiment-wide sanity checks; no missing batches, no mismatching processor configuration, no missed BEs, no other running executors, and no other DBMS processes. 


\begin{tabular}{|l|l|c|}
\hline 1 & Number of Missing Batches & 0 \\
\hline 2 & Number of Inconsistent Processor Configuration Violations & 0 \\
\hline 3 & Number of Missed Batch Executions & 0 \\
\hline 4 & Number of Other Executor Violations & 0 \\
\hline 5 & Number of Other DBMS Process Violations & 0 \\
\hline
\end{tabular}

Table 5: Experiment-Wide Sanity Checks in the Confirmatory Evaluation

\begin{tabular}{|l|l|l|}
\hline 6 & Percentage of Zero TPS & $0.036 \%(25 / 70000)$ \\
\hline 7 & Percentage of Connection Time Limit Violations & $3.5 \%(2434 / 70000)$ \\
\hline 8 & Percentage of Abnormal ATPT & $0.97 \%(679 / 70000)$ \\
\hline
\end{tabular}

Table 6: Batch Execution Sanity Checks in the Confirmatory Evaluation

\begin{tabular}{|l|l|l|}
\hline 9 & Percentage of Transient Thrashing & $0 \%(0 / 1400)$ \\
\hline
\end{tabular}

Table 7: A Batchset Instance Sanity Check in the Confirmatory Evaluation

In the BE sanity checks shown in Table 6 , we had $0.036 \%$ zero TPS violations, $3.5 \%$ connection time limit violations, and $0.97 \%$ abnormal ATP violations. These rates were very low. As exhibited in Table 7 , we had no violations of (9) concerning the batchset sanity check. Our experiment data passed all of these sanity checks.

At Step 2 we drop the BEs identified as violation in Table 7. Table 8 shows how many BEs were valid at the beginning of Step 2 and after Step 2: about $4.39 \%$ BEs were dropped. Table 8 also exhibits how many BSIs were dropped after each sub-step in Step 3. As indicated by the last row of Step 2, interestingly we had no dropped BSIs when starting Step 3; every BSI survived. At Step 3-(i) we dropped BSIs that did not have ten batches. There were 61 BSIs dropped at this step. Step 3-(ii) dropped BSIs revealing transient thrashing at Step 1. Here there was no need to drop BSIs, as indicated by Table 7 . Step 3-(iii) again dropped BSIs revealing transient thrashing among the remaining BSIs until this step. No BSIs were dropped. In sum, we dropped in concert about $4.4 \%$ BSIs throughout Step 3.

At Step 4, we computed the TP for each of the retained BSIs in the same way as done with the exploratory experiment. We detected thrashing in a total of 482 samples, consisting of 148/334 samples from the read/update groups. The percentage of the thrashing batchsets varied decreased to $36 \%$ from $49 \%$ in the exploratory experiment. 


\begin{tabular}{|l|c|}
\hline At Start of Step 2 & 70,000 BEs \\
At Start of Step 2 & 1,400 BSIs \\
\hline After Step 2 & 66,927 BEs (4.4\% dropped) \\
\hline \hline At Start of Step 3 & 1,400 BSIs (0\% dropped) \\
\hline After Step 3-(i) & 1,339 BSIs \\
\hline After Step 3-(ii) & 1,339 BSIs \\
\hline After Step 3-(iii) & 1,339 BSIs (4.4\% dropped) \\
\hline
\end{tabular}

Table 8: The Number of Batch Executions and Batchset Instances after Each SubStep

We reaffirmed in the confirmatory experiment that 1) every DBMS showed thrashing, and 2) there were still many BSIs (about one-thirds (149)) for which the DBMSes experienced early thrashing, that is, under MPL 300, in the confirmatory experiment.

\subsection{Testing Assumptions of Multi-Linear Regression}

Before fitting the model via multiple linear regression (MLR), we need to check several assumptions required by MLR. One key assumption is independence of residuals. This assumption expects that the residuals should be uncorrelated serially from one sample to the next; namely, the level of error should be independent of when a sample is collected (time dependency). (Note that patternless, random residuals imply independence of errors.)

The assumption was tested via the Durbin-Watson (D-W) statistic [55]. We employed durbinWatsonTest() in R [54] to test the assumption against our experimental data. The resulting D-W scores of TP were 1.4 and 1.1 for the read and update groups. For the data to not indicate auto-correlation, we need a score of two. But the computed scores indicated some type of positive correlation among the residuals in our data. We also computed the D-W scores of ATPT but still observed such a positive correlation.

Indeed, this violation was somewhat expected, in that 1) a limited number (e.g, only five) of DBMS subjects were used in our experiment, and 2) the overall violation results from only one (or at most two) of the DBMSes that had violating samples. To further look into this violation, we also calculated the per-DBMS D-W statistics for TP. As a result, we identified several possible issues, including 1) per-DBMS low sample size, 2) some DBMSes revealing auto-correlated samples, and 3) clustered TP and ATPT across DBMSes. Nevertheless, in general the violation did not invalidate the model [55]. (In future work more samples per DBMS are needed and then checked to see if 
the D-W test can be passed at an individual DBMS and the overall level. If this violation persists in bigger sample sizes, a correction using the CochraneOrcutt estimation method [56] may be applied.)

Another key assumption is homoscedasticity. This assumption tests whether the variance of residuals is constant along the line of the best fit. ncvTest() of $\mathrm{R}$ was used for testing this assumption. The $p$-values of the test were 0.20 (0.47), greater than 0.05 , for the read (update) group, which satisfied the homoscedasticity assumption.

The third key assumption is multicollinearity. This assumption tested is whether two or more independent variables are highly correlated with each other. vif() of $\mathrm{R}$ yielded variance inflation factors (VIFs) on the data. The outcome of vif() showed that none of our independent variables had the square root of its corresponding VIF higher than 2, meaning no high correlation of the independent variables. Our data thus satisfied the multicollinearity assumption.

The next key assumption is no outliers. We can test by using Cook's Distance (CD) [57]. We used cooks . distance() of R to test this assumption. The data did not show any significant outliers because the CD values were fewer than 1.

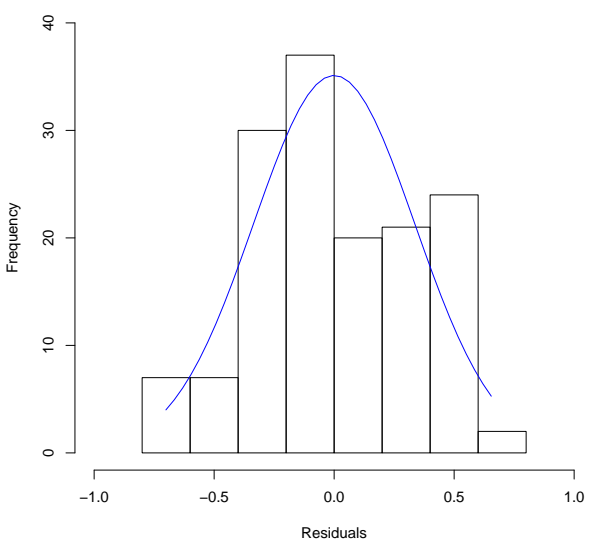

(a) Read-only Batchset Group

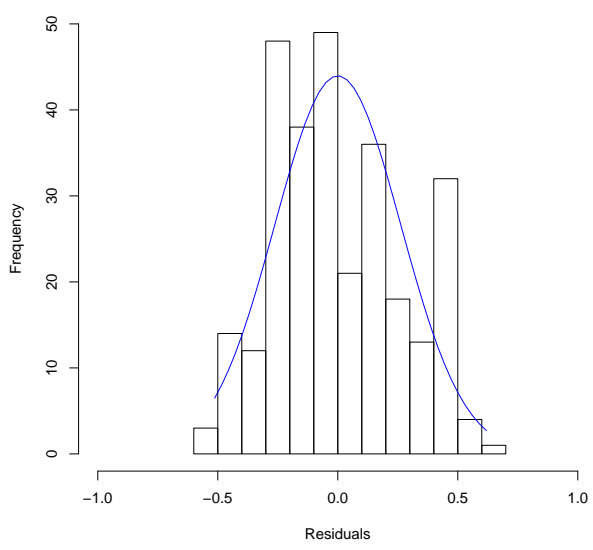

(b) Update-only Batchset Group

Figure 7: Testing Normality of Residuals on the Confirm. Eval. Data

The last key assumption is (5) normality of residuals. This assumption 


\begin{tabular}{c|l|l} 
Variable & $T P$ & ATPT \\
\hline numProcs & H1: $\mathbf{0 . 3 1}^{*}$ & H2: $\mathbf{- 0 . 3 2}$ \\
\hline ATPT & H3: $\mathbf{- 0 . 2 6}$ & - \\
\hline PK & H4: $\mathbf{0 . 2 1}^{*}$ & H5: $\mathbf{- 0 . 5 5 *}$ \\
\hline
\end{tabular}

Table 9: Testing Hypotheses 1-5: Correlations on the Read Group (supported hypotheses marked in bold, and (*): significant at 0.05 level)

indicates that the residuals should be approximately normally distributed; that is, the observed and predicted values should come from the same distribution [58]. A model satisfying this assumption is expected to predict values higher than actual and lower than actual with equal probability.

The assumption was tested by obtaining the histogram of the residuals and superimposing a normal curve on that histogram for each group as shown in Figure 7. The residuals of our data appeared to follow very approximate normality.

All things considered, our experimental data appear to satisfy the MLR assumptions.

\subsection{Confirmatory Evaluation Results}

We now evaluate the refined model in Figure 6.

\subsubsection{Correlational Analysis}

We conducted the confirmatory pair-wise correlational analysis on the refined model.

Table 9 exhibits the computed correlation factor associated with each hypothesis on the read group. All the hypotheses (H1-H5) were supported and significant. The actual levels of $\mathrm{H} 1$ and $\mathrm{H} 5$ were consistent with our predictions in Table 4. The actual levels of $\mathrm{H} 2, \mathrm{H} 3$, and $\mathrm{H} 4$ were medium, low, and low while the predicted levels of low, medium, and medium, respectively. Note that the actual and predicted levels were very close. Such a minor difference was acceptable. Thus, the direct correlations of the read model were empirically confirmed by these correlational analysis results.

Table 10 shows the computed correlation factor associated with each hypothesis for the update group. As for the read group, all the hypotheses (H1-H3) for the update group were supported and significant. The actual levels were consistent with the predicted levels by the model. Therefore, the 


\begin{tabular}{c|l|l} 
Variable & $T P$ & $A T P T$ \\
\hline numProcs & H1: $\mathbf{- 0 . 3 6}^{*}$ & H2: $\mathbf{- 0 . 1 6}$ \\
\hline ATPT & H3: $\mathbf{- 0 . 1 3}^{*}$ & - \\
\hline
\end{tabular}

Table 10: Testing Hypotheses 1-3: Correlations on the Update Group (supported hypotheses marked in bold, and '*': significant at 0.05 level)

direct correlations of the update model were also empirically confirmed by the correlational analysis results shown in Table 10.

\subsubsection{Regression Analysis}

We performed a regression over the independent variables that predict DBMS thrashing (TP variable). Specifically, we computed the overall fit of the refined model for the read and update groups. For the read group our model explained $14 \%$ of the variance of the dependent variable of DBMS thrashing. For the update group our model explained $15 \%$ of the variance of DBMS thrashing. These regression results show that the refined model could to some extent successfully explain TP variance.

We also performed a regression on average transaction processing time (ATPT variable). The refined model explained $29 \%$ of the variance for ATPT on the read group. The fit of the refined model was the same as that of the initial model. We found that in the confirmatory experiment most of the DBMSes were consistent with the direction of the correlations of numProcs and PK with ATPT. We suspect that such an agreement contributed to the high variance explained.

Turning to the update group, the amount of variance explained was $8 \%$. As a check, we computed the variance explained by the initial model in the confirmatory experiment: the explained variance was $12 \%$. We attribute the decrease to the variables and associations removed from the initial model.

\subsubsection{Causal Mediation Analysis}

We conducted causal mediation analysis on the refined model, as was done with the exploratory evaluation. For the read group, we reaffirmed the statistical significance of the mediating effects of PK and numProcs via ATPT on the TP. For the update group, we also verified the statistical significance of the mediating effect of numProcs via ATPT on TP. These results demonstrate that the mediation via ATPT on TP in the refined model was empirically 
verified for each group.

\subsubsection{Path Analysis}

We performed the path analysis for the refined model in Figure 6, to determine what paths in the model were statistically significant, using the path coefficients from the regression outputs of the read and update model fits. We computed the total (indirect + direct) effect on each path in the model.

Regarding the read group, all the paths were significant as shown in Figure 6(a). The direct effect of PK to ATPT $(-0.42)$ was bigger than that of numProcs to ATPT $(-0.34)$. In addition, the total effect of numProcs on $\mathrm{TP}$ was $0.44(=0.34+(-0.34 \times(-0.28)))$, which was greater than that of PK $(0.38=0.26+(-0.42 \times(-0.28)))$ and that of ATPT on TP $(-0.28)$.

For the read group, PK and numProcs were the most significant factors on ATPT and TP in the model, respectively. In the confirmatory experiment we added one more value (or, six processors) in the operationalization of numProcs. This perhaps may have contributed to the increased significance. More investigations about the increase are needed. That said, we reaffirm that numProcs and PK had positive correlations with TP, and ATPT also had a negative correlation with TP.

Concerning the update model, all the paths were significant, as illustrated in Figure 6(b). The total effect of numProcs to TP was $-0.37+(-0.34 \times$ $(-0.13))=-0.33$, which was stronger than that of ATPT to TP $(-0.13)$. For the update group, it was numProcs that showed the most significant factor on both ATPT and TP. We reassert that numProcs and ATPT had negative correlations with TP in the refined model.

In Figure 6, the error variances of ATPT $\left(e_{a t p t}\right)$ and TP $\left(e_{t p}\right)$ were 0.84 and 0.93 for the read group and 0.96 and 0.92 for the update group, each

computed by $\sqrt{\left(1-R_{a t p t}^{2}\right)}$ and $\sqrt{\left(1-R_{t p}^{2}\right)}$. There is much room to improve the model, such as exploring and then examining more variables and associations to further explain the thrashing variance. That said, our model is a contribution, as it has already identified the significance of several variables (that is, the number of processors, presence of primary keys, and average transaction processing time) and their associations that have a definite impact on thrashing.

Lastly, the overall fits of the read and update path models were estimated as follows. 
Fit for the read model $=1-e_{a t p t}^{2} \times e_{t p}^{2}=1-0.84^{2} \cdot 0.93^{2}=0.39$

Fit for the update model $=1-e_{\text {atpt }}^{2} \times e_{t p}^{2}=1-0.96^{2} \cdot 0.92^{2}=0.23$

The fit (0.39) of the read model is higher than that (0.23) of the update model. This difference was primarily because the variance explained by ATPT was higher in the read model than in the update model. To increase the fit of the update model, the amount of variance explained by ATPT should be improved by looking into other possible variables and their correlations that have significant impact on ATPT.

The estimated fits also indicate that the update model has more room for improvement than the read model does. As mentioned above, it would be advantageous to explore other variables and investigate their causal relationships on TP. Again, our model provides a good basis for such variable and correlation exploration.

In sum, all the confirmatory evaluation results provide empirical support for the novel structural causal model of DBMS thrashing in this article that we introduce here.

\section{Engineering Implications}

While developing the refined model through a series of large-scale experiments managed by AZDBLAB over about a year, we uncovered several surprising results that provide system-context indications as to how modern transaction processing can be further improved.

- Every DBMS used in our experiment exhibited thrashing. Previous studies $[8,23]$ pointed out that some open-source DBMSes such as PostgreSQL and MySQL experienced thrashing. We show that proprietary DBMSes are also vulnerable to thrashing.

- Thrashing in DBMSes occurred extensively over increasing MPLs. In particular, some DBMSes experienced early thrashing, under an MPL of 300. It was surprising to see that DBMSes were sometimes overwhelmed by very simple read-only (update-only) including just select and projection (update) clauses. 
- Lastly, a heuristic nature of DBMSes seemed related to the presence of thrashing. One thing to notice was that a DBMS does not always thrash on the same batchset. For instance, for a particular batchset the DBMSes thrashing in the exploratory experiment but not in the confirmatory experiment, and vice versa. Another was that given a certain batchset, thrashing was experienced by some DBMSes but not by others; namely, every DBMS did not experience thrashing on the same batchset. We found out that query optimizers of some of the DBMSes revealed a heuristic nature of execution plan selection. We therefore suspect that the occurrence of thrashing is associated with such a heuristic.

These results suggest that research on thrashing should be pursued in conjunction with multi-core architectures. Fortunately, the causal model in Figure 6 helps point out specifically where that research might be focused.

Our model implies some engineering implications for DBAs and for DBMS researchers and developers, for better understanding and properly coping with thrashing. (These implications are complementary to the practical lessons already given in a previous literature [59].)

1. For read-only workloads, we have the following general advice.

- DBAs should enable multi-core processors. DBMSes can utilize their parallel processing ability when serving the workloads. In our experiment DBMSes showed increased TP (thrashing point) as more processors were available. DBAs thus may consider exposing to DBMSes as many processors as available.

- DBAs should continue to utilize primary keys whenever possible. We have observed in our experiment that although workloads were increased, DBMSes were still scalable, resulting from their primary index made available by primary keys. We thus can infer that reducing I/O overhead can be a critical factor in preventing transaction throughput from falling off at a lower MPL. DBAs therefore may consider several approaches to improve I/O, including 1) increasing physical memory, 2) creating secondary indexes, 3) enabling caches from disk drive through file system up to DBMS buffer, and 4) replacing traditional disks with solid-state drives (SSDs) for reducing access latency [60]. These approaches 
can delay thrashing, or perhaps even prevent it in some situations. In future work the effectiveness of each of these approaches could be evaluated.

- DBAs should pay attention to response time of transactions. Another key factor in increasing TP was to shorten transaction response time. Our experiment showed that TP decreased as ATPT increased. When DBMS thrashing occurs, DBAs should check if the response time of transactions in their workloads sharply rose at a certain point. To reduce the response time, we suggest using as many processors as possible and specifying primary keys on the tables referenced by the transactions, as mentioned above. We challenge database researchers to identify other ways of improving the response time.

- DBAs should be aware that the thrashing phenomenon tends to be DBMS-specific. As mentioned before, we observed that even if the same batchset was presented to all the DBMSes under the same condition, one DBMS may thrash whereas another didn't. This implies that DBAs should check their specific transaction management and tuning parameters such as the maximum number of connections and size of shared buffer.

2. When treating update-only workloads, we advise the following.

- Using many processors may not help increase TP for update-only workloads. We observed in our experiment that as the number of processors increased, TP decreased, which was different from what was observed in the read-only transactions. When treating concurrent update-only transactions, DBMSes are charged with substantial lock management overhead on the same objects referenced by the transactions. When multi-core processors are employed, DBMSes may struggle to tolerate inter-processor contention on the synchronization constructs related to exclusive lock management. DBAs thus should be aware of processor contention caused by more provisioning, when serving update-only workloads.

- When thrashing is observed, DBAs are encouraged to examine whether the response time of transactions sharply went up at a certain point. As in the case of the read-only workloads, we observed that decreasing ATPT could help increase TP in update- 
only workloads as well. Although we did not see the significance of primary keys on ATPT in our data, we still think that I/O could be one of the influential factors in decreasing response time. As far as the significance of $\mathrm{I} / \mathrm{O}$ to reduce response time in update-only transactions is concerned, more research is needed.

- Increasing the number of processors may improve ATPT. In our experiment DBMSes sped up processing the update-only transactions as the number of processors increased. Multi-core processors may enable intra-parallelism within the same transaction, contributing to decreasing response time. However, it was not helpful to have more processors in increasing TP, as mentioned in the first bullet above. DBAs therefore should attempt to make more processors available to DBMSes to reduce transaction response time. Future work should address what other alternatives can improve response time, resulting in increasing TP. 


\section{Conclusions and Future Work}

DBMS thrashing is an impediment to application performance. The less thrashing that occurs, the better. For that reason, it is important to understand this phenomenon, so that it can be predicted and possibly prevented.

We have explored many possible candidate factors affecting the thrashing phenomenon that are present in modern relational DBMSes. We presented a refinement of a structural causal model to explain the variance of thrashing, which we evaluated with substantial empirical data that manipulated the underlying factors.

The refined model of the read group explained about $14 \%$ and $29 \%$ of the variances of TP (thrashing point) and average transaction processing time (ATPT), respectively. TP has statistically significant correlations with ATPT, numProcs, and PK, among which numProcs is the most significant to TP. ATPT has significant correlations with numProcs and PK, and PK is more significant to ATPT. The model also reveals significant mediations from numProcs and PK through ATPT to TP.

The refined model of the update group explained about $15 \%(8 \%)$ of the variances of TP (ATPT). TP has statistically significant correlations with numProcs and ATPT, and numProcs is more significant to TP. ATPT has a significant correlation with numProcs, the only significant factor to ATPT in the model. The model shows a significant mediation from numProcs through ATPT to TP as well.

The refined model suggests several engineering implications that can be helpful to DBAs and provide research questions to database researchers. An important implication of the model is that transaction response time is one of the most significant factors of thrashing. When thrashing is detected, DBAs should examine whether the response time of transactions in their workloads suddenly increased at a certain point. The model also suggests that decreasing the response time can increase TP. To reduce the response time of read-only workloads, the DBAs should consider increasing the number of processors and specifying a primary key for every table. To speed up the processing time of update-only workloads, the DBAs may consider using increasing the number of processors. As increasing the number of processors has a negative correlation with TP, however, it would be helpful to to explore other ways of decreasing the response time of the update-only workloads.

To the best of our knowledge, our thrashing causal model is the first to be articulated across multiple relational DBMSes. Our model leaves room for 
elaboration, as there are surely other unknown origins of DBMS thrashing.

We suggest the following areas where future work would be fruitful: (i) consider different types - compute-bound, mixed, nested, multi-level, chained, queued, and distributed - of transactions, (ii) refine the operationalization of ROSE and SF and reexamine their significance on TP and ATPT in the read and update groups, (iii) expand the range or number of parameter values for various operationalizations, (iv) consider in more detail moderated mediations that were not statistically significant, (v) re-investigate the significance of PK on ATPT and TP in the update group, (vi) scrutinize the cause of the increased significance of numProcs for the read group in relation to the added value in the numProcs operationalization, (vii) apply the alternatives for improving I/O, mentioned in Section 10, to investigate their relative efficacy, (viii) explore other alternatives to reduce transaction response time in the update group, (ix) consider the impact of insertions and deletions and more complicated transactions over multiple tables/columns, and lastly, (x) collect more samples from DBMSes and re-performing the $\mathrm{D}-\mathrm{W}$ test against the data.

The model we have articulated explains about 14\% (15\%) of the DBMS thrashing variance for read-only (update-only) transactional workload. Note that in general high variance explained is exceedingly hard to obtain in many disciplines, as many factors can make a discernible increase in the explained variance. Our results imply much room for further refinement and elaboration. Specifically, the database community can propose further refinements to the refined model to hopefully increase its explanatory power, by (i) studying the effects of a yet to be proposed causal factors: different types of indexes or short transaction (selecting a single row only) rates in a batch, (ii) exploring a wider variety of DBMS subjects, (iii) exploring unknown relationships between the variables in the final model, (iv) looking into the impact of a variety of predicates involving joins, IN, and aggregates beyond simple range scans, (v) investigating whether thrashing can also be attributed to contention on the pool of threads handling active transactions, (vi) incorporating MPL as a (dependent) variable into the model and operationalizing it by measuring the number of sessions (potentially pooled across a limited number of active connections) as a representative of the fire hose approach [61], and finally, (vii) considering other statistical tools, such as nonlinear regression [62], logistic regression [63], and structural equation modeling [64], for the model.

In all cases, our model is the appropriate starting place for an elaborated causal model with more factors. 


\section{Acknowledgments}

This work was supported in part by the National Science Foundation under grants IIS-0639106, IIS-1016205, IIS-0415101, and EIA-0080123, as well as by the EDISON project managed by the National Research Foundation of Korea (NRF), funded by the Ministry of Science, ICT \& Future Planning (NRF-2011-0020576). We thank John Kececioglu, Peter J. Downey, and anonymous reviewers for their insightful comments to enhance the quality of this article. We also appreciate Tom Lowry and Tom Buchanan for maintaining our experimental instrument, a laboratory of ten machines and associated software.

\section{References}

[1] T. Horikawa, An Approach for Scalability-Bottleneck Solution: Identification and Elimination of Scalability Bottlenecks in a DBMS, SIGSOFT Software Engineering Notes (SEN) 36 (2011) 31-42.

[2] S. Chaudhuri, U. Dayal, An Overview of Data Warehousing and OLAP Technology, SIGMOD Record 26 (1997) 65-74.

[3] S. A. Schuster, Relational Data Base Management for On-Line Transaction Processing, Technical Report 81.5, Tandem Computers Incorporated, 1981.

[4] R. Johnson, I. Pandis, A. Ailamaki, Critical Sections: Re-emerging Scalability Concerns for Database Storage Engines, in: DaMoN '08, 2008, pp. $35-40$.

[5] P. J. Denning, Thrashing, http://denninginstitute.com/pjd/PUBS/ ENC/thrash08.pdf, viewed March 31, 2014.

[6] G. Weikum, A. Möenkeberg, C. Hasse, P. Zabback, Self-Tuning Database Technology and Information Services: From Wishful Thinking to Viable Engineering, in: VLDB '02, 2002, pp. 20-31.

[7] R. Johnson, I. Pandis, N. Hardavellas, A. Ailamaki, B. Falsafi, Shore-MT: A Scalable Storage Manager for the Multicore Era, in: EDBT '09, 2009, pp. 24-35. 
[8] H. Jung, H. Han, A. D. Fekete, G. Heiser, H. Y. Yeom, A Scalable Lock Manager for Multicores, in: SIGMOD '13, 2013, pp. 73-84.

[9] A. Thomasian, Concurrency Control: Methods, Performance, and Analysis, ACM Computing Surveys 30 (1998) 70-119.

[10] B. Mozafari, C. Curino, A. Jindal, S. Madden, Performance and Resource Modeling in Highly-Concurrent OLTP Workloads, in: SIGMOD '13, 2013, pp. 301-312.

[11] S. Currim, S. Ram, A. Durcikova, F. Currim, Using a Knowledge Learning Framework to Predict Errors in Database Design, Information Systems 40 (2014) 11-31.

[12] A. Dan, D. M. Dias, P. S. Yu, The Effect of Skewed Data Access on Buffer Hits and Data Contention in a Data Sharing Environment, in: VLDB '90, Morgan Kaufmann Publishers Inc., 1990, pp. 419-431.

[13] A. Dan, P. S. Yu, J. Y. Chung, Characterization of Database Access Pattern for Analytic Prediction of Buffer Hit Probability, The VLDB Journal (VLDBJ) 4 (1995) 127-154.

[14] M. J. Carey, S. Krishnamurthi, M. Livny, Load Control for Locking: The 'Half-and-Half' Approach, in: PODS '90, 1990, pp. 72-84.

[15] A. Mönkeberg, G. Weikum, Conflict-Driven Load Control for the Avoidance of Data-Contention Thrashing, in: ICDE '91, 1991, pp. 632-639.

[16] A. Mönkeberg, G. Weikum, Performance Evaluation of an Adaptive and Robust Load Control Method for the Avoidance of Data-Contention Thrashing, in: VLDB '92, 1992, pp. 432-443.

[17] Y. C. Tay, N. Goodman, R. Suri, Locking Performance in Centralized Databases, ACM TODS 10 (1985) 415-462.

[18] A. Thomasian, Thrashing in Two-Phase Locking Revisited, in: ICDE '92, IEEE, 1992, pp. 518-526.

[19] A. Thomasian, A Two-Phase Locking Performance and Its Thrashing Behavior, ACM TODS 18 (1993) 579-625. 
[20] A. Thomasian, A Performance Comparison of Locking Methods with Limited Wait Depth, IEEE TKDE 9 (1997) 421-434.

[21] P. S. Yu, D. M. Dias, S. S. Lavenberg, On the Analytical Modeling of Database Concurrency Control, J. ACM 40 (1993) 831-872.

[22] P. A. Franaszek, J. T. Robinson, A. Thomasian, Concurrency Control for High Contention Environments, ACM TODS 17 (1992) 304-345.

[23] T. Horikawa, Latch-Free Data Structures for DBMS: Design, Implementation, and Evaluation, in: SIGMOD '13, 2013, pp. 409-420.

[24] B. Zhang, M. Hsu, Modeling Performance Impact of Hot Spots, in: Performance of Concurrency Control Mechanisms in Centralized Database Systems, Prentice-Hall, Inc., 1995, pp. 148-165.

[25] A. F. Hayes, Introduction to Mediation, Moderation, and Conditional Process Analysis: A Regression-Based Approach, Guilford, 2013.

[26] E. J. Pedhazur, Multiple Regression in Behavioral Research, Thomson Learning, 1997.

[27] K. Imai, L. Keele, D. Tingley, A General Approach to Causal Mediation Analysis, Psychological Methods 15 (2010) 309-334.

[28] Y.-K. Suh, R. T. Snodgrass, R. Zhang, AZDBLab: A Lab Information System for Large-scale Empirical DBMS Studies, PVLDB 7 (2014) $1641-1644$.

[29] A. Thomasian, Chapter 56: Performance Evaluation of Computer Systems, in: Computing Handbook, Third Edition, Chapman and Hall/CRC 2014, 2014, pp. 1-50.

[30] S. S. Lavenberg, Computer Performance Modeling Handbook, Academic Press, 1983.

[31] D. T. McWherter, B. Schroeder, A. Ailamaki, M. Harchol-Balter, Priority Mechanisms for OLTP and Transactional Web Applications, in: ICDE '04, 2004, pp. 535-546. 
[32] J. Letchner, M. Balazinska, C. Ré, M. Philipose, Approximation Trade-offs in a Markovian Stream Warehouse: An Empirical Study, Information Systems 39 (2014) 290-304.

[33] M. Döhring, H. A. Reijers, S. Smirnov, Configuration vs. Adaptation for Business Process Variant Maintenance: An Empirical Study, Information Systems 39 (2014) 108-133.

[34] M. Zheng, J. Tucek, D. Huang, F. Qin, M. Lillibridge, E. S. Yang, B. W. Zhao, S. Singh, Torturing Databases for Fun and Profit, in: OSDI '14, 2014, pp. 449-464.

[35] J. Gray, The Transaction Concept: Virtues and Limitations (Invited Paper), in: VLDB '81, 1981, pp. 144-154.

[36] P. Atzeni, F. Bugiotti, L. Rossi, Uniform Access to NoSQL Systems, Information Systems 43 (2014) 117-133.

[37] R. Cattell, Scalable SQL and NoSQL Data Stores, SIGMOD Record 39 (2011) 12-27.

[38] FAL Labs, Tokyo Cabinet: A Modern Implementation of DBM, http: //fallabs.com/tokyocabinet/, viewed February 7, 2015.

[39] Symas Corporation, Symas Lightning Memory-Mapped Database (LMDB), http://symas.com/mdb/, viewed February 7, 2015.

[40] Hwaci, SQLite, http://www.sqlite.org/, viewed February 7, 2015.

[41] MariaDB Foundation, MariaDB: An Enhanced, Drop-in Replacement for MySQL, https://mariadb.org/, viewed February 7, 2015.

[42] R. T. Snodgrass, P. Denning, The Science of Computer Science: Closing Statement: The Science of Computer Science (Ubiquity Symposium), Ubiquity 2014 (2014) 1-11.

[43] J. Gray, A. Reuter, Transaction Processing: Concepts and Techniques, 1st ed., Morgan Kaufmann Publishers Inc., 1992.

[44] Oracle Corporation, The Java Database Connectivity (JDBC), viewed April 15, 2014. 
[45] P. G. Selinger, M. M. Astrahan, D. D. Chamberlin, R. A. Lorie, T. G. Price, Access Path Selection in a Relational Database Management System, in: SIGMOD '79, 1979, pp. 23-34.

[46] K. J. Preacher, D. D. Rucker, A. F. Hayes, Addressing Moderated Mediation Hypotheses: Theory, Methods, and Prescriptions, Multivariate Behavioral Research 42 (2007) 185-227.

[47] Y.-K. Suh, Exploring Causal Factors of DBMS Thrashing, Ph.D. dissertation, Dept. of Computer Science, Univ. of Arizona, 2015.

[48] I. Pandis, R. Johnson, N. Hardavellas, A. Ailamaki, Data-oriented Transaction Execution, PVLDB 3 (2010) 928-939.

[49] J. Nilsson, F. Dahlgren, Improving Performance of Load-store Sequences for Transaction Processing Workloads on Multiprocessors, in: ICPP '99, IEEE, 1999, pp. 246-255.

[50] Joint Committee for Guides in Metrology, International Vocabulary of Metrology Basic and General Concepts and Associated Terms (VIM) (3rd Ed.), viewed December 05, 2014.

[51] S. Currim, R. T. Snodgrass, Y.-K. Suh, R. Zhang, M. Johnson, C. Yi, DBMS Metrology: Measuring Query Time, in: Proceedings of the 39th ACM SIGMOD International Conference on Management of Data (SIGMOD '13), ACM, 2013, pp. 261-272.

[52] S. Currim, R. T. Snodgrass, Y.-K. Suh, R. Zhang, A Better Way of Measuring Query Time, 2015. Under review.

[53] S. Currim, R. T. Snodgrass, Y.-K. Suh, R. Zhang, A Causal Model of DBMS Suboptimality, 2016. Under review.

[54] R Core Team, R: A Language and Environment for Statistical Computing, R Foundation for Statistical Computing, 2014.

[55] J. Durbin, G. S. Watson, Testing for Serial Correlation in Least Squares Regression, Biometrika 58 (1971) 1-19. 
[56] D. Cochrane, G. H. Orcutt, Application of Least Squares Regression to Relationships Containing Auto-Correlated Error Terms, Journal of the American Statistical Association 44 (1949) 32-61.

[57] R. D. Cook, Detection of Influential Observations in Linear Regression, Technometrics 19 (1977) 15-18.

[58] Wikiversity, Multiple Linear Regression/Assumptions, http: //en.wikiversity.org/wiki/Multiple_linear_regression/ Assumptions, viewed October 31, 2014.

[59] V. Holt, M. Ramage, K. Kear, N. W. Heap, The Usage of Best Practices and Procedures in the Database Community, Information Systems 49 (2015) 163-181.

[60] Y.-K. Suh, B. Moon, A. Efrat, J.-S. Kim, S.-W. Lee, Memory Efficient and Scalable Address Mapping for Flash Storage Devices, Journal of Systems Architecture 60 (2014) 357-371.

[61] T. Barclay, J. Gray, D. Slutz, Microsoft TerraServer: A Spatial Data Warehouse, in: Proceedings of the 2000 ACM SIGMOD International Conference on Management of Data (SIGMOD '00), ACM, New York, NY, USA, 2000, pp. 307-318.

[62] T. Amemiya, Nonlinear Regression Models, Handbook of Econometrics 1 (1983) 333-389.

[63] J. S. Long, Regression Models for Categorical and Limited Dependent Variables, 2 ed., SAGE Publications, 1997.

[64] J. Ullman, Structural Equation Modeling: Reviewing the Basics and Moving Forward, Journal of Personality Assessment 87 (2006) 35-50. 